Homology, Homotopy and Applications, vol.20(2), 2018, pp.289-313

\title{
SELF-MAPS OF SPACES IN FIBRATIONS
}

\author{
NOBUYUKI ODA AND TOSHIHIRO YAMAGUCHI
}

(communicated by Donald M. Davis)

\begin{abstract}
Self-maps of spaces in fibrations which appear in Postnikov systems are studied. The results are applied to obtain relations among self-homotopy equivalences of spaces in those fibrations to prove inequalities among self-closeness numbers of those spaces. Making use of the models of Sullivan, self-closeness numbers of rational spaces are also examined and some examples of rational spaces are studied precisely.
\end{abstract}

\section{Introduction}

The purpose of this paper is to study self-homotopy equivalences of spaces in connection with fibrations and obtain some relations between self-homotopy equivalences of a space and those of the induced fiber. Therefore, our results can be applied to determine the self-closeness number $N \mathcal{E}(X)$ of a space $X$ defined by Choi and Lee [2] (see Definition 3.2).

Let $G$ be an abelian group and $K(G, n)$ the Eilenberg-MacLane space. In this paper we consider the following type of fibration sequence:

$$
\cdots \longrightarrow \Omega Y \stackrel{\Omega \gamma}{\longrightarrow} \Omega K(G, m+2)=K(G, m+1) \stackrel{q}{\longrightarrow} X \stackrel{i}{\longrightarrow} Y \stackrel{\gamma}{\longrightarrow} K(G, m+2),
$$

where $m \geqslant 0$ and $Y$ is a 0 -connected CW-complex such that $\pi_{d}(Y)=0$ for $d \geqslant m+1$. In [15] we studied self-homotopy equivalences of spaces in cofibrations. However, the results in this paper are not obtained by trivial dual arguments.

In Section 2 we prove the following (Theorem 2.6): Assume that there exist maps $f: Y \rightarrow Y, g: X \rightarrow X$ and $\Omega s: \Omega K(G, m+2) \rightarrow \Omega K(G, m+2)$ such that the left and the middle squares are homotopy commutative in the following diagram:

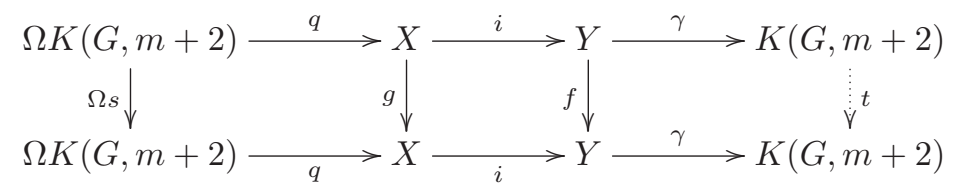

Then there exists an element $t \in[K(G, m+2), K(G, m+2)]$ such that the right square in the above diagram is homotopy commutative and $s=a \circ t \circ b$ for some

The first author was partly supported by JSPS KAKENHI Grant Number JP15K04884.

Received February 6, 2018, revised April 5, 2018; published on June 27, 2018.

2010 Mathematics Subject Classification: 55P10, 55P62, 55Q05, 55R05.

Key words and phrases: self-homotopy equivalence, fibration, self-closeness number, Sullivan model.

Article available at http://dx.doi.org/10.4310/HHA.2018.v20.n2.a15

Copyright (C) 2018, International Press. Permission to copy for private use granted. 
$a, b \in \mathcal{E}(K(G, m+2))$. Here, the symbol $[X, Y]$ is the set of homotopy classes of the maps $f: X \rightarrow Y$ for spaces $X$ and $Y$ and $\mathcal{E}(X)$ is the set of homotopy classes of self-homotopy equivalences of a space $X$.

In Section 3 we consider the relations of $N \mathcal{E}(Y)$ and $N \mathcal{E}(X)$. Assume that

$$
\gamma_{\sharp}([Y, Y]) \subset \gamma^{\sharp}([K(G, m+2), K(G, m+2)]) .
$$

Then we show that $N \mathcal{E}(Y) \leqslant N \mathcal{E}(X) \leqslant m+1$ (Theorem 3.3). Moreover, (Theorem 3.9) if the induced homomorphism

$$
\gamma^{\sharp}:[K(G, m+2), K(G, m+2)] \rightarrow[Y, K(G, m+2)]
$$

is a monomorphism and satisfies the condition that

$$
\gamma_{\sharp}(\mathcal{E}(Y)) \subset \gamma^{\sharp}([K(G, m+2), K(G, m+2)]),
$$

then the inequality $N \mathcal{E}(X) \leqslant N \mathcal{E}(Y)$ holds. It follows that (Theorem 3.10) if the induced homomorphism $\gamma^{\sharp}:[K(G, m+2), K(G, m+2)] \rightarrow[Y, K(G, m+2)]$ is an isomorphism, then $N \mathcal{E}(X)=N \mathcal{E}(Y)$. Furthermore, in Proposition 3.13 and Theorem 3.18, we consider more precisely the cases where the groups $G$ are some special groups and obtain some sufficient conditions for $N \mathcal{E}(X)=N \mathcal{E}(Y)$.

In Section 4 we use Sullivan models to obtain rational results. Making use of the algebraic method, we can study fibrations. First, we give some interesting examples and counter-examples. In particular, we show that there can exist six different self-closeness numbers' types of almost free circle actions on a product of five odd dimensional spheres in Example 4.6. Second, we prove the following (Theorem 4.8): Let $m \geqslant 2$ and $K(\mathbb{Z}, m+1) \stackrel{q}{\rightarrow} X \stackrel{i}{\rightarrow} Y \stackrel{\gamma}{\rightarrow} K(\mathbb{Z}, m+2)$ be a fibration sequence such that $Y$ is 1 -connected and $\pi_{t}(Y)=0$ for $t \geqslant m+1$. If $H^{m+2}(Y ; \mathbb{Z})$ is torsion-free and $\gamma \neq 0$, then $N \mathcal{E}(X) \leqslant N \mathcal{E}(Y)$. It is the result of Theorem 3.9 in the case $G=\mathbb{Z}$ under a different condition than the above. Third, we show a dichotomy example (Theorem 4.10) of fibrations for the self-closeness number, that is, we characterize the conditions $N \mathcal{E}\left(X_{0}\right)<N \mathcal{E}\left(Y_{0}\right)$ and $N \mathcal{E}\left(X_{0}\right)=N \mathcal{E}\left(Y_{0}\right)$ by the other conditions such as formality $[\mathbf{3}]$ and cohomological conditions. Here $X_{0}$ and $Y_{0}$ are the rationalizations of $X$ and $Y$, respectively.

\section{An application of a result of Ganea}

In this paper, we consider based spaces and based maps and based homotopies. The symbol $f \simeq g: X \rightarrow Y$ is a based homotopy relation and $[f]: X \rightarrow Y$ is the homotopy class of $f$ and $[X, Y]$ is the set of homotopy classes $[f]: X \rightarrow Y$. However, in many cases, the homotopy class $[f]$ and the map $f$ are not distinguished for simplicity. For a map $f: X \rightarrow Y$, we denote the induced functions between homotopy sets by $f_{\sharp}:[A, X] \rightarrow[A, Y]$ and $f^{\sharp}:[Y, Z] \rightarrow[X, Z]$ for any spaces $A$ and $Z$; the induced homomorphisms between homology groups by $f_{*}: H_{*}(X ; G) \rightarrow H_{*}(Y ; G)$ and those between cohomology groups by $f^{*}: H^{*}(Y ; G) \rightarrow H^{*}(X ; G)$ for any abelian groups $G$.

We study a dual result of Proposition 2 of [15] by the form of Proposition 2.5 and 
Theorem 2.6: Consider the following diagram for $m \geqslant 0$ :

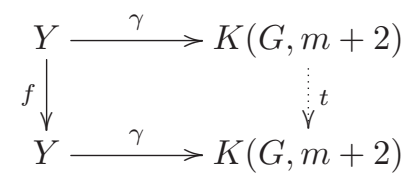

There does not always exist a map $t: K(G, m+2) \rightarrow K(G, m+2)$ which makes the above diagram homotopy commutative (Remark 2.7). However, for a fibration $X \stackrel{i}{\rightarrow}$ $Y \stackrel{\gamma}{\rightarrow} K(G, m+2)$, we prove by Proposition 2.5 that if two maps $g$ and $f$ exist such that the left square in the following diagram is homotopy commutative, then, there exists a map $t: K(G, m+2) \rightarrow K(G, m+2)$ which makes the right square homotopy commutative:

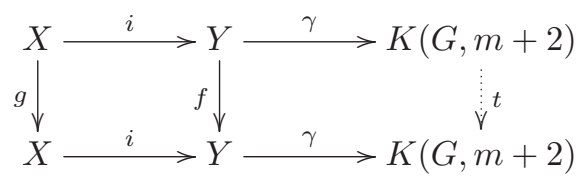

Therefore, Theorem 2.6 is not a complete dual of Proposition 2 of [15]. In the proof of Proposition 2 of [15], we used the properties of cofibrations and we applied Hurewicz theorem to the results of homology groups. However, we cannot use the dual arguments for fibrations and therefore we use Theorem 1.1 of Ganea [6] in the following arguments.

Let $X \stackrel{i}{\rightarrow} Y \stackrel{\gamma}{\rightarrow} K(G, m+2)$ be a fibration. Let $\bar{\gamma}: Y \cup_{i} C X \rightarrow K(G, m+2)$ be an extension of $\gamma: Y \rightarrow K(G, m+2)$ and let $F_{\bar{\gamma}}$ be the homotopy fibre of $\bar{\gamma}: Y \cup_{i} C X \rightarrow$ $K(G, m+2)$ as in the following commutative diagram:

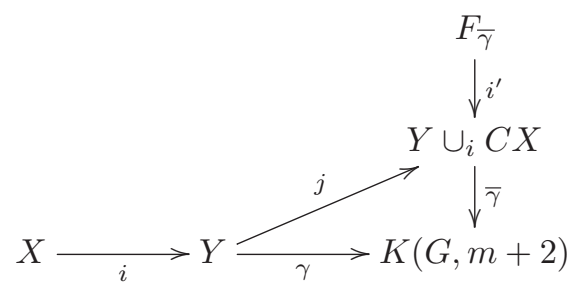

Then the homotopy fibre $F_{\bar{\gamma}}$ of $\bar{\gamma}$ is weakly homotopy equivalent to $X * \Omega K(G, m+2)$ by Theorem 1.1 of Ganea $[\mathbf{6}]$.

Lemma 2.1. Let $m, n \geqslant 0$. If $X$ is n-connected, then $F_{\bar{\gamma}} \simeq X * \Omega K(G, m+2)$ is $(n+m+2)$-connected.

Proof. Since $F_{\bar{\gamma}} \simeq X * \Omega K(G, m+2) \simeq(\Sigma X) \wedge \Omega K(G, m+2)$, we have the result.

Proposition 2.2. Let $Y$ be 0 -connected in the fibration $X \stackrel{i}{\rightarrow} Y \stackrel{\gamma}{\rightarrow} K(G, m+2)$. Then

(1) $\bar{\gamma}_{\sharp}: \pi_{k}\left(Y \cup_{i} C X\right) \rightarrow \pi_{k}(K(G, m+2))$ is surjective for $k \leqslant m+3$ and injective for $k \leqslant m+2$.

(2) $\bar{\gamma}_{*}: H_{k}\left(Y \cup_{i} C X ; G\right) \rightarrow H_{k}(K(G, m+2) ; G)$ is surjective for $k \leqslant m+3$ and injective for $k \leqslant m+2$. 
(3) $\bar{\gamma}^{*}: H^{k}(K(G, m+2) ; G) \rightarrow H^{k}\left(Y \cup_{i} C X ; G\right)$ is injective for $k \leqslant m+3$ and surjective for $k \leqslant m+2$.

Proof. (1) We have the result by Lemma 2.1 and the long homotopy exact sequence for the fibration

$$
F_{\bar{\gamma}} \stackrel{i^{\prime}}{\longrightarrow} Y \cup_{i} C X \stackrel{\bar{\gamma}}{\longrightarrow} K(G, m+2) .
$$

(2) Part (1) implies that $\bar{\gamma}_{*}: H_{k}\left(Y \cup_{i} C X ; \mathbb{Z}\right) \rightarrow H_{k}(K(G, m+2) ; \mathbb{Z})$ is surjective for $k \leqslant m+3$ and injective for $k \leqslant m+2$ by a theorem of J. H. C. Whitehead, where $\mathbb{Z}$ is the additive group of integers (see (7.13) Theorem (p. 181) of Whitehead [18]). Hence we have the result by the universal coefficient theorem for homology (see Theorem 55.1, Corollary 55.2 and Lemma 54.3 of Munkres [13]).

(3) We have the result by the universal coefficient theorem for cohomology (see Corollary 53.2 and Exercise 4 (p. 319) of Munkres [13]).

Remark 2.3. The results of Proposition 2.2 is also obtained by applying the result of Exercise 23 (p. 155) of Gray [7].

Proposition 2.4. Let $m \geqslant 0$. Assume that $Y$ is 0 -connected. Then

$$
\begin{aligned}
{\left[Y \cup_{i} C X, K(G, m+2)\right] } & =\{a \circ \bar{\gamma} \mid a \in[K(G, m+2), K(G, m+2)]\} \\
& \cong[K(G, m+2), K(G, m+2)] .
\end{aligned}
$$

Proof. By Proposition 2.2(3) we have an isomorphism

$$
\bar{\gamma}^{*}: H^{m+2}(K(G, m+2) ; G) \rightarrow H^{m+2}\left(Y \cup_{i} C X ; G\right),
$$

which is an isomorphism between homotopy sets:

$$
\bar{\gamma}^{\sharp}:[K(G, m+2), K(G, m+2)] \rightarrow\left[Y \cup_{i} C X, K(G, m+2)\right] .
$$

Proposition 2.5. Let $m \geqslant 0$ and let $Y$ be 0 -connected. Let $X \stackrel{i}{\rightarrow} Y \stackrel{\gamma}{\rightarrow} K(G, m+2)$ be a fibration. Assume that the left square in the following diagram is homotopy commutative, namely $f \circ i \simeq i \circ g$. Then, there exists a map $t: K(G, m+2) \rightarrow K(G, m+2)$ which makes the middle and the right squares homotopy commutative in the following diagram, where $\bar{\gamma} \circ j=\gamma$ :

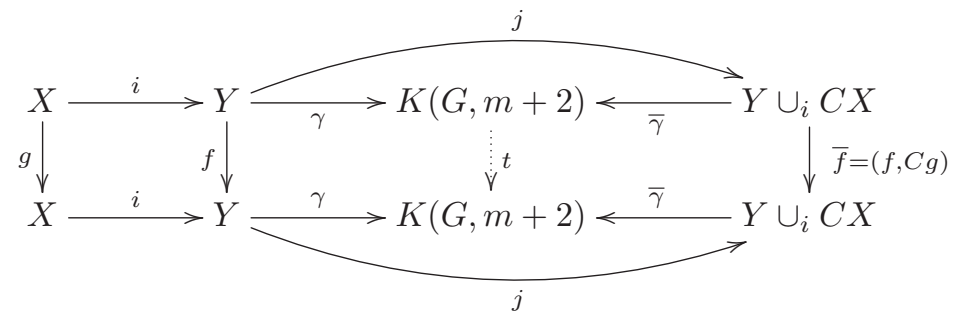

Proof. By Proposition 2.4, we have a map $t: K(G, m+2) \rightarrow K(G, m+2)$ which makes the right square homotopy commutative. It follows that the middle square is homotopy commutative too:

$$
t \circ \gamma=t \circ \bar{\gamma} \circ j=\bar{\gamma} \circ \bar{f} \circ j=\bar{\gamma} \circ j \circ f=\gamma \circ f .
$$


Theorem 2.6. Let $m \geqslant 0$ and let $Y$ be 0 -connected and $\pi_{d}(Y)=0$ for $d \geqslant m+1$. Let $X \stackrel{i}{\rightarrow} Y \stackrel{\gamma}{\rightarrow} K(G, m+2)$ be a fibration. Assume that there exist maps $f: Y \rightarrow Y$, $g: X \rightarrow X$ and $\Omega s: \Omega K(G, m+2) \rightarrow \Omega K(G, m+2)$ such that the left and the middle squares are homotopy commutative in the following diagram:

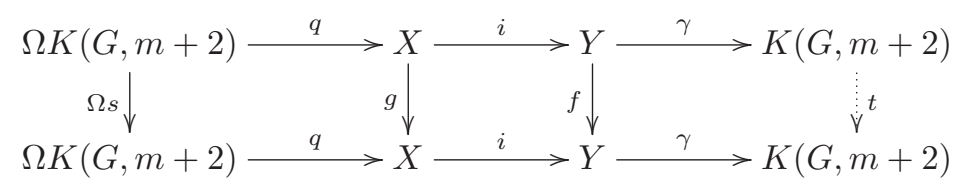

Then there exists an element $t \in[K(G, m+2), K(G, m+2)]$ such that the right square in the above diagram is homotopy commutative and the relation $s=a \circ t \circ b$ holds for some $a, b \in \mathcal{E}(K(G, m+2))$.

Proof. Consider the following diagram of pairs of spaces:

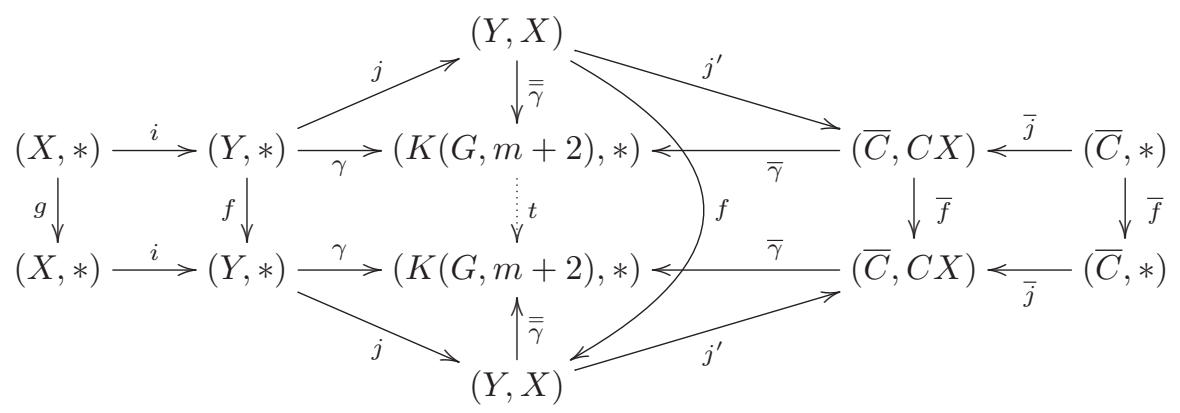

where $\bar{C}=Y \cup_{i} C X$ and $\bar{f}=f \cup C g: \bar{C}=Y \cup_{i} C X \rightarrow Y \cup_{i} C X=\bar{C}$ is the extension of $f: Y \rightarrow Y$. Moreover, $j, j^{\prime}$ and $\bar{j}$ are inclusions and

$$
\overline{\bar{\gamma}}=\bar{\gamma} \circ j^{\prime}:(Y, X) \rightarrow(\bar{C}, C X) \rightarrow(K(G, m+2), *)
$$

by definition and the map $f: Y \rightarrow Y$ is also written by $f:(Y, *) \rightarrow(Y, *)$ and $f:(Y, X) \rightarrow(Y, X)$. There exists a map $t: K(G, m+2) \rightarrow K(G, m+2)$ which makes the above diagram homotopy commutative by Proposition 2.5. Then we have the following homotopy commutative diagram:

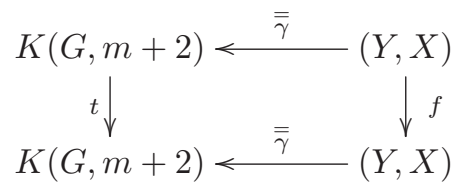

Therefore the following diagram of homotopy groups is commutative:

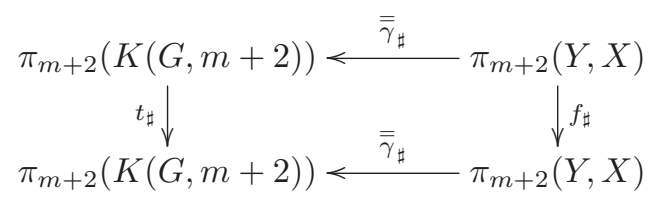

Here, we see $\overline{\bar{\gamma}}_{\sharp}: \pi_{m+2}(Y, X) \rightarrow \pi_{m+2}(K(G, m+2)) \cong G$ is an isomorphism by the property of the fibration. Moreover, we have isomorphisms $\partial: \pi_{m+2}(Y, X) \rightarrow \pi_{m+1}(X)$ 
and $q_{\sharp}: \pi_{m+1}(\Omega K(G, m+2)) \rightarrow \pi_{m+1}(X)$, since $\pi_{d}(Y)=0$ for $d \geqslant m+1$. Then we have the result by the following commutative diagram, since we may write

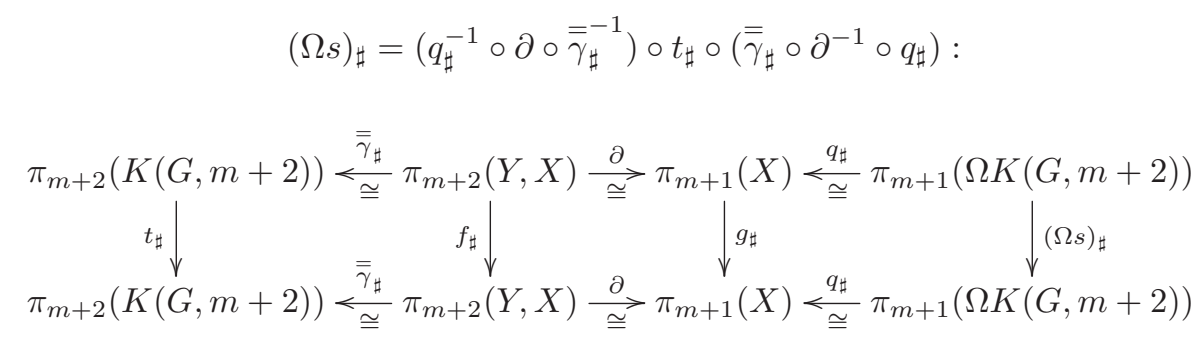

Remark 2.7. Let $m \geqslant 0$. Consider the following diagram:

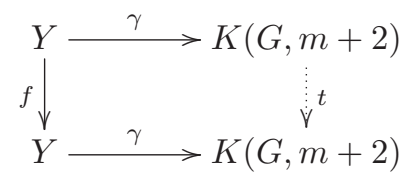

(1) There does not always exist a map $t: K(G, m+2) \rightarrow K(G, m+2)$ which makes the above diagram homotopy commutative as in the following example:

Let $p_{1}: Y=S^{m+2} \times S^{m+2} \rightarrow S^{m+2}$ be the projection to the first factor and $i: S^{m+2} \subset K(\mathbb{Z}, m+2)$ the inclusion map. Let $\gamma$ be the composite:

$$
\gamma=i \circ p_{1}: Y=S^{m+2} \times S^{m+2} \rightarrow S^{m+2} \subset K(\mathbb{Z}, m+2) .
$$

If $\tau: S^{m+2} \times S^{m+2} \rightarrow S^{m+2} \times S^{m+2}$ is the switching map, then the following diagram is not homotopy commutative for any map $t: K(\mathbb{Z}, m+2) \rightarrow K(\mathbb{Z}, m+2)$ :

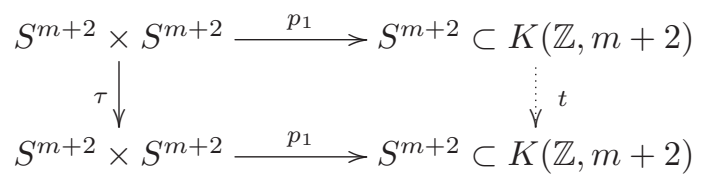

We note that

$$
\begin{aligned}
{\left[S^{m+2} \times S^{m+2}, K(\mathbb{Z}, m+2)\right] } & \cong\left[S^{m+2} \vee S^{m+2}, K(\mathbb{Z}, m+2)\right] \\
& \cong\left[S^{m+2}, K(\mathbb{Z}, m+2)\right] \oplus\left[S^{m+2}, K(\mathbb{Z}, m+2)\right] \cong \mathbb{Z} \oplus \mathbb{Z} .
\end{aligned}
$$

(2) If $[Y, K(G, m+2)]=\{a \circ \gamma \mid a \in[K(G, m+2), K(G, m+2)]\}$, then there exists a map $t: K(G, m+2) \rightarrow K(G, m+2)$ which makes the above diagram homotopy commutative.

Example 2.8 (Rational version). Let $m \geqslant 2$. Let $\mathbb{Q}$ be the additive group of rational numbers. We consider the following fibration sequence:

$$
\cdots \longrightarrow \Omega Y \stackrel{\Omega \gamma}{\longrightarrow} \Omega K(\mathbb{Q}, m+2)=K(\mathbb{Q}, m+1) \stackrel{q}{\longrightarrow} X \stackrel{i}{\longrightarrow} Y \stackrel{\gamma}{\longrightarrow} K(\mathbb{Q}, m+2) .
$$

Assume that $Y$ is 1-connected and $\pi_{d}(Y)=0$ for $d \geqslant m+1$. Let $f: Y \rightarrow Y$ and $g: X \rightarrow X$ be maps such that $f \circ i \simeq i \circ g$. Assume that there exists an element 
$\Omega s \in[\Omega K(\mathbb{Q}, m+2), \Omega K(\mathbb{Q}, m+2)] \cong \mathbb{Q}$ such that the left square is homotopy commutative in the following diagram:

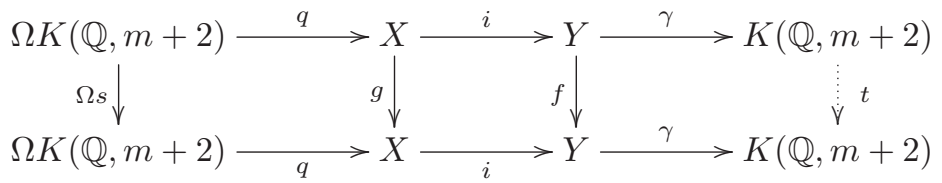

Then there exists an element $t \in[K(\mathbb{Q}, m+2), K(\mathbb{Q}, m+2)] \cong \mathbb{Q}$ such that the right square in the above diagram is homotopy commutative and $s=a \circ t \circ b$ for some $a, b \in \mathcal{E}(K(\mathbb{Q}, m+2))$ by Theorem 2.6 applied to the case $G=\mathbb{Q}$.

\section{A theorem for special types of fibrations}

We first consider the following fibration sequence:

$$
\cdots \longrightarrow \Omega Y \stackrel{\Omega \gamma}{\longrightarrow} \Omega K(G, m+2)=K(G, m+1) \stackrel{q}{\longrightarrow} X \stackrel{i}{\longrightarrow} Y \stackrel{\gamma}{\longrightarrow} K(G, m+2),
$$

where $G$ is an abelian group.

Proposition 3.1. Let $m \geqslant 0$. Assume that $\pi_{d}(Y)=0$ for $d \geqslant m+1$ in the following fibration sequence:

$$
\cdots \longrightarrow \Omega Y \stackrel{\Omega \gamma}{\longrightarrow} K(G, m+1) \stackrel{q}{\longrightarrow} X \stackrel{i}{\longrightarrow} Y \stackrel{\gamma}{\longrightarrow} K(G, m+2) .
$$

Then the following results hold:

$$
\begin{cases}\pi_{d}(X)=0 & \text { if } d \geqslant m+2, \\ q_{\sharp}: G=\pi_{m+1}(K(G, m+1)) \rightarrow \pi_{m+1}(X) & \text { is an isomorphism, } \\ i_{\sharp}: \pi_{d}(X) \rightarrow \pi_{d}(Y) \text { is an isomorphism } & \text { if } d \leqslant m .\end{cases}
$$

Proof. We have the results by the long homotopy exact sequence.

3.1. A condition for $N \mathcal{E}(Y) \leqslant N \mathcal{E}(X)$

Choi and Lee $[\mathbf{2}]$ introduced the following concept:

Definition 3.2. For any space $X$, the subset $\mathcal{A}_{\sharp}^{k}(X)$ of $[X, X]$ is defined by $\mathcal{A}_{\sharp}^{k}(X)=\left\{f \in[X, X] \mid f_{\sharp}: \pi_{i}(X) \stackrel{\cong}{\longrightarrow} \pi_{i}(X)\right.$ is an isomorphism for any $\left.i \leqslant k\right\}$, and the self-closeness number $N \mathcal{E}(X)$ of $X$ by

$$
N \mathcal{E}(X)=\min \left\{k \mid \mathcal{A}_{\sharp}^{k}(X)=\mathcal{E}(X)\right\} .
$$

Theorem 3.3. Let $m \geqslant 0$. Let $Y$ be a 0 -connected $C W$-complex with $\pi_{d}(Y)=0$ for $d \geqslant m+1$ in the following fibration sequence:

$$
\cdots \longrightarrow \Omega Y \stackrel{\Omega \gamma}{\longrightarrow} \Omega K(G, m+2)=K(G, m+1) \stackrel{q}{\longrightarrow} X \stackrel{i}{\longrightarrow} Y \stackrel{\gamma}{\longrightarrow} K(G, m+2) .
$$

If $\gamma_{\sharp}([Y, Y]) \subset \gamma^{\sharp}([K(G, m+2), K(G, m+2)])$, then $N \mathcal{E}(Y) \leqslant N \mathcal{E}(X) \leqslant m+1$.

Proof. If $N \mathcal{E}(X) \geqslant m$, then $N \mathcal{E}(X) \geqslant N \mathcal{E}(Y)$ holds, since $N \mathcal{E}(Y) \leqslant m$ by the Whitehead theorem (see (3.9) Corollary (p. 222) of Whitehead [18]). 
Assume that $N \mathcal{E}(X)=k<m$. Let $f: Y \rightarrow Y$ be a map which satisfies the condition that

$$
f_{\sharp}: \pi_{d}(Y) \rightarrow \pi_{d}(Y)
$$

is an isomorphism for any $d \leqslant k$.

By the assumption $\gamma_{\sharp}([Y, Y]) \subset \gamma^{\sharp}([K(G, m+2), K(G, m+2)])$, there exists a map $s: K(G, m+2) \rightarrow K(G, m+2)$ which makes the right square in the following diagram homotopy commutative:

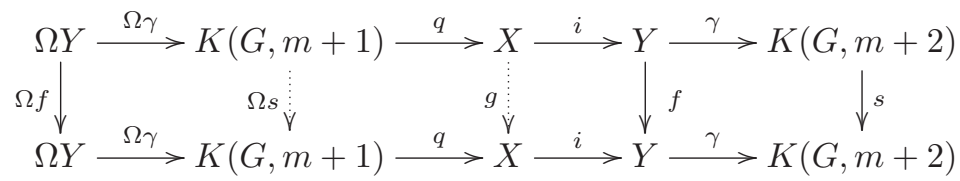

Therefore, there exists a map $g: X \rightarrow X$ which makes the above diagram homotopy commutative. We see $g_{\sharp}: \pi_{d}(X) \rightarrow \pi_{d}(X)$ is an isomorphism for any $d \leqslant k$, since $i_{\sharp}: \pi_{d}(X) \rightarrow \pi_{d}(Y)$ is an isomorphism for any $d \leqslant m$ by Proposition 3.1 and $f_{\sharp}: \pi_{d}(Y) \rightarrow \pi_{d}(Y)$ is an isomorphism for any $d \leqslant k$. It follows that $g \in \mathcal{E}(X)$ since $N \mathcal{E}(X)=k$. Hence the map $\Omega s: K(G, m+1) \rightarrow K(G, m+1)$ is a homotopy equivalence, since $q_{\sharp}: \pi_{m+1}(K(G, m+1)) \rightarrow \pi_{m+1}(X)$ is an isomorphism. Therefore, $f \in$ $\mathcal{E}(Y)$ because $f: Y \rightarrow Y$ is a weak homotopy equivalence by the long homotopy exact sequence and the five lemma, and hence $N \mathcal{E}(Y) \leqslant k=N \mathcal{E}(X)$.

Proposition 3.4. Let $m \geqslant 0$. Assume that $Y$ is a 0 -connected $C W$-complex and $\pi_{d}(Y)=0$ for $d \geqslant m+1$ in the following fibration sequence:

$$
\cdots \longrightarrow \Omega Y \stackrel{\Omega \gamma}{\longrightarrow} \Omega K(G, m+2)=K(G, m+1) \stackrel{q}{\longrightarrow} X \stackrel{i}{\longrightarrow} Y \stackrel{\gamma}{\longrightarrow} K(G, m+2) .
$$

Assume that there exists an element $\zeta \in[Y, K(G, m+2)]$ such that

$$
[Y, K(G, m+2)]=\{a \circ \zeta \mid a \in[K(G, m+2), K(G, m+2)]\} .
$$

If $a \circ b=b \circ a$ for any $a, b \in[K(G, m+2), K(G, m+2)]$, then

$$
N \mathcal{E}(Y) \leqslant N \mathcal{E}(X) \leqslant m+1 \text {. }
$$

Proof. Let $f \in[Y, Y]$ be any element. Since we may write $\gamma=c \circ \zeta$ for some $c$ of $[K(G, m+2), K(G, m+2)]$ by our assumption, we see $\gamma \circ f=c \circ \zeta \circ f$ and $\zeta \circ f$ is in $[Y, K(G, m+2)]$. Hence, there exists an element $s \in[K(G, m+2), K(G, m+2)]$ such that $\zeta \circ f=s \circ \zeta$. It follows that

$$
\gamma \circ f=c \circ \zeta \circ f=c \circ s \circ \zeta=s \circ c \circ \zeta=s \circ \gamma \text {. }
$$

Therefore the condition $\gamma_{\sharp}([Y, Y]) \subset \gamma^{\sharp}([K(G, m+2), K(G, m+2)])$ in Theorem 3.3 holds, and hence we have the result.

Remark 3.5. The difference between $N \mathcal{E}(Y)$ and $N \mathcal{E}(X)$ in Theorem 3.3 can be arbitrarily large as in the following example: Let $m \geqslant 2$. If $Y=K(\mathbb{Z}, 2)$ and $\gamma=0$, the constant map, in Theorem 3.3, then $X=Y \times K(G, m+1)$ and we have

$$
N \mathcal{E}(Y)=2<m+1=N \mathcal{E}(X) .
$$


Example 3.6. Let $Y=\mathbb{C} P^{\infty} \vee \mathbb{C} P^{\infty}$. Then,

$$
\begin{aligned}
& {\left[\mathbb{C} P^{\infty} \vee \mathbb{C} P^{\infty}, K(\mathbb{Z}, 4)\right] \cong H^{4}\left(\mathbb{C} P^{\infty} \vee \mathbb{C} P^{\infty} ; \mathbb{Z}\right)} \\
& \cong H^{4}\left(\mathbb{C} P^{\infty}\right) \oplus H^{4}\left(\mathbb{C} P^{\infty} ; \mathbb{Z}\right) \cong \mathbb{Z}\left\{x^{2}\right\} \oplus \mathbb{Z}\left\{y^{2}\right\} .
\end{aligned}
$$

Here, $H^{4}\left(\mathbb{C} P^{\infty} ; \mathbb{Z}\right) \cong \mathbb{Z}\left\{x^{2}\right\}$ and $H^{4}\left(\mathbb{C} P^{\infty} ; \mathbb{Z}\right) \cong \mathbb{Z}\left\{y^{2}\right\}$ with $\operatorname{deg}(x)=\operatorname{deg}(y)=2$.

On the other hand, we see $[K(\mathbb{Z}, 4), K(\mathbb{Z}, 4)] \cong H^{4}(K(\mathbb{Z}, 4) ; \mathbb{Z}) \cong \mathbb{Z}$. Therefore,

$$
[Y, K(\mathbb{Z}, 4)]=\left[\mathbb{C} P^{\infty} \vee \mathbb{C} P^{\infty}, K(\mathbb{Z}, 4)\right] \neq\{a \circ \zeta \mid a \in[K(\mathbb{Z}, 4), K(\mathbb{Z}, 4)]\},
$$

for any $\zeta \in[Y, K(\mathbb{Z}, 4)]$.

\subsection{A condition for $N \mathcal{E}(X) \leqslant N \mathcal{E}(Y)$}

Theorem 3.7. Let $m \geqslant 0$. Let $Y$ be a 0 -connected $C W$-complex with $\pi_{d}(Y)=0$ for $d \geqslant m+1$ in the following fibration sequence:

$$
\Omega K(G, m+2)=K(G, m+1) \stackrel{q}{\longrightarrow} X \stackrel{i}{\longrightarrow} Y \stackrel{\gamma}{\longrightarrow} K(G, m+2) .
$$

Assume that $\gamma: Y \rightarrow K(G, m+2)$ satisfies the condition that if the following diagram:

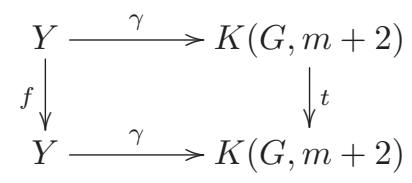

is homotopy commutative for some $f \in \mathcal{E}(Y)$ and $t: K(G, m+2) \rightarrow K(G, m+2)$, then $t \in \mathcal{E}(K(G, m+2))$. Under this condition the following inequality holds:

$$
N \mathcal{E}(X) \leqslant N \mathcal{E}(Y)(\leqslant m) .
$$

Proof. Assume that $N \mathcal{E}(Y)=k(\leqslant m)$. Let $g: X \rightarrow X$ be a map which satisfies

$$
g_{\sharp}: \pi_{d}(X) \rightarrow \pi_{d}(X)
$$

is an isomorphism for any $d \leqslant k \leqslant m$. By the argument in Nomura [14], there exist maps $f: Y \rightarrow Y$ and $s: K(G, m+2) \rightarrow K(G, m+2)$ which make the following diagram homotopy commutative:

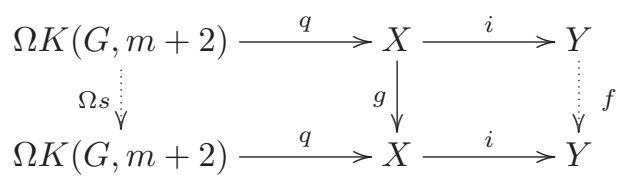

We see $f_{\sharp}: \pi_{d}(Y) \rightarrow \pi_{d}(Y)$ is an isomorphism for any $d \leqslant k$, since $i_{\sharp}: \pi_{d}(X) \rightarrow \pi_{d}(Y)$ is an isomorphism for any $d \leqslant m$ by Proposition 3.1 and $g_{\sharp}: \pi_{d}(X) \rightarrow \pi_{d}(X)$ is an isomorphism for any $d \leqslant k$. Hence $f \in \mathcal{E}(Y)$ since $N \mathcal{E}(Y)=k$.

Now, by Theorem 2.6, there exists a map $t: K(G, m+2) \rightarrow K(G, m+2)$ such that $s=a \circ t \circ b$ for some $a, b \in \mathcal{E}(K(G, m+2))$ and the diagram

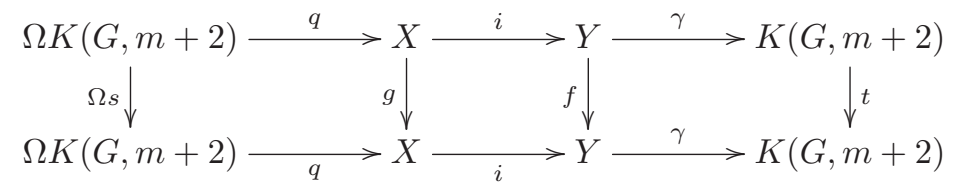


is homotopy commutative. Since $f \in \mathcal{E}(Y)$, we have $t \in \mathcal{E}(K(G, m+2))$ by the assumption. Therefore $g \in \mathcal{E}(X)$ by the five lemma applied to the long homotopy exact sequences. It follows that $N \mathcal{E}(X) \leqslant k=N \mathcal{E}(Y)$.

Proposition 3.8. Let $m \geqslant 0$. Assume that $Y$ is a 0 -connected $C W$-complex in the following fibration sequence:

$$
\Omega K(G, m+2) \stackrel{q}{\longrightarrow} X \stackrel{i}{\longrightarrow} Y \stackrel{\gamma}{\longrightarrow} K(G, m+2) .
$$

Assume that the induced homomorphism

$$
\gamma^{\sharp}:[K(G, m+2), K(G, m+2)] \rightarrow[Y, K(G, m+2)]
$$

is a monomorphism and satisfies the condition that

$$
\gamma_{\sharp}(\mathcal{E}(Y)) \subset \gamma^{\sharp}([K(G, m+2), K(G, m+2)]) .
$$

Let $f: Y \rightarrow Y$ and $t: K(G, m+2) \rightarrow K(G, m+2)$ be maps and the following diagram homotopy commutative:

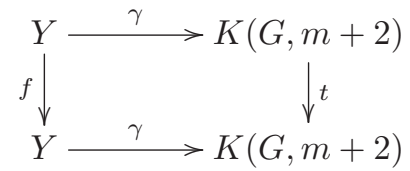

Then, $f \in \mathcal{E}(Y)$ implies $t \in \mathcal{E}(K(G, m+2))$.

Proof. Let $f \in \mathcal{E}(Y)$. Let $\bar{f} \in \mathcal{E}(Y)$ be the homotopy inverse of $f$, namely $f \circ \bar{f} \simeq$ $1_{Y} \simeq \bar{f} \circ f$. We see $\gamma \circ \bar{f}=\bar{t} \circ \gamma$ for an element $\bar{t} \in[K(G, m+2), K(G, m+2)]$ by our assumption that $\gamma_{\sharp}(\mathcal{E}(Y)) \subset \gamma^{\sharp}([K(G, m+2), K(G, m+2)])$ as in the following diagram:

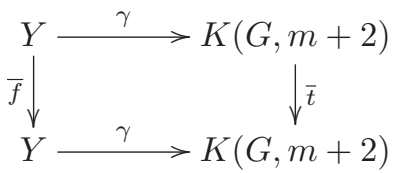

We see

$$
\begin{aligned}
& 1_{K(G, m+2)} \circ \gamma=\gamma \circ 1_{Y}=\gamma \circ f \circ \bar{f}=t \circ \gamma \circ \bar{f}=t \circ \bar{t} \circ \gamma ; \\
& 1_{K(G, m+2)} \circ \gamma=\gamma \circ 1_{Y}=\gamma \circ \bar{f} \circ f=\bar{t} \circ \gamma \circ f=\bar{t} \circ t \circ \gamma .
\end{aligned}
$$

Since $\gamma^{\sharp}:[K(G, m+2), K(G, m+2)] \rightarrow[Y, K(G, m+2)]$ is a monomorphism, we have $t \circ \bar{t} \simeq 1_{K(G, m+2)} \simeq \bar{t} \circ t$ and hence $t \in \mathcal{E}(K(G, m+2))$.

Theorem 3.9. Let $m \geqslant 0$. Let $Y$ be a 0 -connected $C W$-complex with $\pi_{d}(Y)=0$ for $d \geqslant m+1$ in the following fibration sequence:

$$
\Omega K(G, m+2)=K(G, m+1) \stackrel{q}{\longrightarrow} X \stackrel{i}{\longrightarrow} Y \stackrel{\gamma}{\longrightarrow} K(G, m+2) .
$$

If the induced homomorphism

$$
\gamma^{\sharp}:[K(G, m+2), K(G, m+2)] \rightarrow[Y, K(G, m+2)]
$$

is a monomorphism and satisfies the condition that

$$
\gamma_{\sharp}(\mathcal{E}(Y)) \subset \gamma^{\sharp}([K(G, m+2), K(G, m+2)]),
$$


then

$$
N \mathcal{E}(X) \leqslant N \mathcal{E}(Y)(\leqslant m) .
$$

Proof. By Theorem 3.7 and Proposition 3.8, we have the result.

Theorem 3.10. Let $m \geqslant 0$. Assume that $X$ and $Y$ are 0 -connected $C W$-complexes and $\pi_{d}(Y)=0$ for $d \geqslant m+1$ in the following fibration sequence:

$$
\Omega K(G, m+2)=K(G, m+1) \stackrel{q}{\longrightarrow} X \stackrel{i}{\longrightarrow} Y \stackrel{\gamma}{\longrightarrow} K(G, m+2) .
$$

If the induced homomorphism

$$
\gamma^{\sharp}:[K(G, m+2), K(G, m+2)] \rightarrow[Y, K(G, m+2)]
$$

is an isomorphism, then $N \mathcal{E}(X)=N \mathcal{E}(Y)$.

Proof. Since $\gamma^{\sharp}:[K(G, m+2), K(G, m+2)] \rightarrow[Y, K(G, m+2)]$ is an isomorphism, the conditions in Theorems 3.3 and 3.9 are satisfied, and hence the result follows.

The following proposition shows a case where the condition of Theorem 3.7 holds.

Proposition 3.11. Let $m \geqslant 0$. Let $t=t 1_{K(\mathbb{Q}, m+2)}: K(\mathbb{Q}, m+2) \rightarrow K(\mathbb{Q}, m+2)$ be a map induced by an element $t \in \mathbb{Q}$. Let $f \in \mathcal{E}(Y)$ and $\bar{f} \in \mathcal{E}(Y)$ be its inverse. Consider the following commutative diagram:

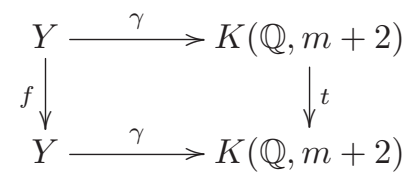

If $\gamma \neq 0$, then $t \neq 0$ and the following diagram is homotopy commutative:

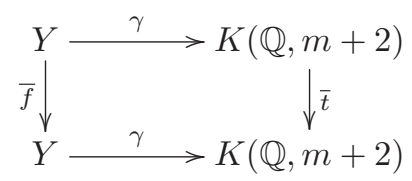

where $\bar{t}=t^{-1}$.

Proof. Since $f \in \mathcal{E}(Y)$, we see $f^{\sharp}:[Y, K(\mathbb{Q}, m+2)] \rightarrow[Y, K(\mathbb{Q}, m+2)]$ is an isomorphism. Therefore, by the commutativity of the above diagram, we see $t \circ \gamma=\gamma \circ f \neq$ 0 , and hence $t \neq 0$. Then we have

$$
\gamma \circ \bar{f} \circ f=\gamma \circ 1_{Y}=1_{K} \circ \gamma=\bar{t} \circ t \circ \gamma=\bar{t} \circ \gamma \circ f .
$$

It follows that

$$
\gamma \circ \bar{f}=\gamma \circ \bar{f} \circ(f \circ \bar{f})=(\gamma \circ \bar{f} \circ f) \circ \bar{f}=(\bar{t} \circ \gamma \circ f) \circ \bar{f}=\bar{t} \circ \gamma \circ(f \circ \bar{f})=\bar{t} \circ \gamma .
$$

Remark 3.12. Let $\mathbb{Z} / \ell$ be the additive group of all residue classes of $\mathbb{Z}$ modulo $\ell$ for some positive integer $\ell \geqslant 2$, that is, $\mathbb{Z} / \ell=\{a+\ell \mathbb{Z} \mid a \in \mathbb{Z}\}$. Let $\mathbb{Z}_{p}^{\wedge}$ be the additive group of $p$-adic integers for some prime number $p$. Let $G=\mathbb{Z}, \mathbb{Q}, \mathbb{Z} / \ell$ or $\mathbb{Z}_{p}^{\wedge}$. For any $c \in G$, we define a homomorphism $h_{c}: G \rightarrow G$ by $h_{c}(x)=c x$ for any $x \in G$. Any homomorphism $h: G \rightarrow G$ can be written $h=h_{c}$ for $c=h(1) \in G$. Moreover, the 
homomorphism $h_{c}$ can be identified with $h(1)=c$ as the multiplication by $c$. Hence we have

$$
\operatorname{Hom}(G, G)=\left\{a 1_{G} \mid a \in G\right\} \cong G \text { for } G=\mathbb{Z}, \mathbb{Q}, \mathbb{Z} / \ell \text { or } \mathbb{Z}_{p}^{\wedge} .
$$

Therefore, for the groups $G=\mathbb{Z}, \mathbb{Q}, \mathbb{Z} / \ell$ or $\mathbb{Z}_{p}^{\wedge}$, we have

$$
\begin{aligned}
{[K(G, m+2),} & K(G, m+2)] \\
& \cong H^{m+2}(K(G, m+2) ; G) \\
& \cong \operatorname{Hom}\left(H_{m+2}(K(G, m+2) ; \mathbb{Z}), G\right) \oplus \operatorname{Ext}\left(H_{m+1}(K(G, m+2) ; \mathbb{Z}), G\right) \\
& \cong \operatorname{Hom}(G, G)=\left\{a 1_{G} \mid a \in G\right\} \cong G .
\end{aligned}
$$

If $G=\mathbb{Z}, \mathbb{Q}, \mathbb{Z} / \ell$ or $\mathbb{Z}_{p}^{\wedge}$, then $a \circ b=b \circ a$ for any $a, b \in[K(G, m+2), K(G, m+2)]$.

Proposition 3.13. Let $G=\mathbb{Z}, \mathbb{Q}, \mathbb{Z} / \ell$ or $\mathbb{Z}_{p}^{\wedge}$ in Theorem 3.9. Assume that there exists an element $\zeta \in[Y, K(G, m+2)]$ which satisfies

$$
[Y, K(G, m+2)]=\{a \circ \zeta \mid a \in[K(G, m+2), K(G, m+2)]\} \cong G .
$$

If $\gamma=c \zeta$ for an element $c \in G$ such that

(i) $G=\mathbb{Z}, \mathbb{Q}$ or $\mathbb{Z}_{p}^{\wedge}$ and $c \neq 0$; or

(ii) $G=\mathbb{Z} / \ell$ with the greatest common divisor $(c, \ell)=1$,

then $N \mathcal{E}(X)=N \mathcal{E}(Y)$.

Proof. We have the results for (i) $G=\mathbb{Q}$ by Theorem 3.10. For (i) $G=\mathbb{Z}$ and $\mathbb{Z}_{p}^{\wedge}$, we see that the conditions in Proposition 3.4 and Theorem 3.9 are satisfied, and hence we have the result.

Now, we consider the case (ii) $G=\mathbb{Z} / \ell$ with $(c, \ell)=1$ : For any $c \in \mathbb{Z} / \ell$, we define a homomorphism $h_{c}: \mathbb{Z} / \ell \rightarrow \mathbb{Z} / \ell$ by $h_{c}(x)=c x$ for any $x \in \mathbb{Z} / \ell$. Any homomorphism $h: \mathbb{Z} / \ell \rightarrow \mathbb{Z} / \ell$ can be written $h=h_{c}$ for some $c \in \mathbb{Z} / \ell$. Then $h_{c}: \mathbb{Z} / \ell \rightarrow \mathbb{Z} / \ell$ is an isomorphism if and only if $(c, \ell)=1$. Hence we have the result by Theorem 3.10 .

The cases where $(c, \ell) \neq 1$ are considered in the next subsection.

\subsection{The case where $G=\mathbb{Z} / \ell$ and $(c, \ell) \neq 1$}

We identify the homomorphism $h_{c}: \mathbb{Z} / \ell \rightarrow \mathbb{Z} / \ell$ with the corresponding map $h_{c}: K(\mathbb{Z} / \ell, m+2) \rightarrow K(\mathbb{Z} / \ell, m+2)$ in the following arguments.

We begin with an example to understand the relation between $\ell$ and $c$.

Example 3.14. We consider the cases $\ell=w \ell^{\prime}, c=w c^{\prime}$ and $\left(c^{\prime}, \ell^{\prime}\right)=1$ in the following:

(1) Let $\ell=2^{4}, w=2$ and $\ell^{\prime}=2^{3}$. If $c^{\prime}=1$, then $c=w c^{\prime}=2$; if $c^{\prime}=3$, then $c=$ $w c^{\prime}=6$; if $c^{\prime}=5$, then $c=w c^{\prime}=10$; if $c^{\prime}=7$, then $c=w c^{\prime}=14$.

(2) Let $\ell=2^{4}, w=4$ and $\ell^{\prime}=2^{2}$. If $c^{\prime}=1$, then $c=w c^{\prime}=4$; if $c^{\prime}=3$, then $c=$ $w c^{\prime}=12$.

To consider the case where $K(G, m+2)=K(\mathbb{Z} / \ell, m+2)$ and $(c, \ell) \neq 1$ in Theorem 3.18, we have to prove the following two lemmas. In Lemma 3.15(1), we consider the case, for example, $\ell=2^{s}, w=2^{t}$ and $\ell^{\prime}=2^{s-t}$ with $1 \leqslant t<s$ and $c^{\prime}$ is an odd integer. 
Lemma 3.15. Let $\ell \geqslant 2$ be an integer and $\ell=w \ell^{\prime}$ and $c=w c^{\prime}$ for some integers $w, c^{\prime}, \ell^{\prime} \geqslant 1$ with $\left(c^{\prime}, \ell^{\prime}\right)=1$. Let $h_{u}, h_{v}: \mathbb{Z} / \ell \rightarrow \mathbb{Z} / \ell$ be homomorphisms for some $u, v \in \mathbb{Z} / \ell$.

(1) Assume that $\ell^{\prime}$ contains any prime divisor of $\ell$. If $h_{c}^{\sharp}\left(h_{u}\right)=h_{c}^{\sharp}\left(h_{v}\right)$ and $(u, \ell)=1$, then $(v, \ell)=1$.

(2) Assume that there exists a prime $p$ such that $(p, w)=p$ and $\left(p, \ell^{\prime}\right)=1$. Then the condition $h_{c}^{\sharp}\left(h_{u}\right)=h_{c}^{\sharp}\left(h_{v}\right)$ and $(u, \ell)=1$ does not always imply $(v, \ell)=1$.

Proof. (1) If $h_{c}^{\sharp}\left(h_{u}\right)=h_{c}^{\sharp}\left(h_{v}\right)$, then $c u=c v$ in $\mathbb{Z} / \ell$ and hence $c u=c v+\ell k$ in $\mathbb{Z}$ for some integer $k$. It follows that $w c^{\prime} u=w c^{\prime} v+w \ell^{\prime} k$ and hence $c^{\prime} u=c^{\prime} v+\ell^{\prime} k$. We see there exist integers $x, y$ such that $\ell^{\prime} x+c^{\prime} y=1$ since $\left(c^{\prime}, \ell^{\prime}\right)=1$. Therefore, the relation $c^{\prime} y u=c^{\prime} y v+\ell^{\prime} y k$ implies $\left(1-\ell^{\prime} x\right) u=\left(1-\ell^{\prime} x\right) v+\ell^{\prime} y k$. Then we have

$$
u=v+\ell^{\prime}(x u-x v+y k) .
$$

Thus the condition $(u, \ell)=1$ implies $(v, \ell)=1$ since $\ell^{\prime}$ contains any prime divisor of $\ell$.

(2) Let $w=p w^{\prime}$ for some integer $w^{\prime} \geqslant 1$ and $\left(p, \ell^{\prime}\right)=1$. Then there exist integers $x$ and $y$ such that $p x+\ell^{\prime} y=1$. Assume that $h_{c}^{\sharp}\left(h_{u}\right)=h_{c}^{\sharp}\left(h_{v}\right)$ and $(u, \ell)=1$. It follows that

$$
c u=c v+\ell k=c v\left(p x+\ell^{\prime} y\right)+\ell k=c v p x+w c^{\prime} v \ell^{\prime} y+\ell k=c(p(v x))+\ell\left(c^{\prime} v y+k\right) .
$$

Hence $c u=c(p(v x))$ in $\mathbb{Z} / \ell$, that is, $h_{c}^{\sharp}\left(h_{u}\right)=h_{c}^{\sharp}\left(h_{p(v x)}\right)$; however, $\ell=w \ell^{\prime}=p w^{\prime} \ell^{\prime}$ and hence $(p(v x), \ell) \neq 1$.

Example 3.16. (1) Let $\ell=n^{s}, c=n^{t}$ for integers $n \geqslant 2$ and $1 \leqslant t<s$. If $c u=c v$ in $\mathbb{Z} / \ell$, then $n^{t} u=n^{t} v+n^{s} k$ in $\mathbb{Z}$ for some integer $k$. It follows that $u=v+n^{s-t} k$. Therefore, $(u, n)=1$ implies $(v, n)=1$.

(2) Let $\ell=6, c=w=2, \ell^{\prime}=3$ so that $\ell=w \ell^{\prime}=2 \cdot 3$. Let $u=5$ and $v=2$. Then $c u=2 \cdot 5=10$ and $c v=2 \cdot 2=4$. Then $c u=c v+1 \cdot \ell$, that is, $h_{c}^{\sharp}\left(h_{u}\right)=c u=$ $c v=h_{c}^{\sharp}\left(h_{v}\right)$ as homomorphisms. However, $h_{u}=h_{5}: \mathbb{Z} / 6 \rightarrow \mathbb{Z} / 6$ is an isomorphism, but $h_{v}=h_{2}: \mathbb{Z} / 6 \rightarrow \mathbb{Z} / 6$ is not an isomorphism. In this case, the condition $h_{c}^{\sharp}\left(h_{u}\right)=h_{c}^{\sharp}\left(h_{v}\right)$ and $(u, \ell)=1$ does not imply $(v, \ell)=1$.

Lemma 3.17. Let $m \geqslant 0$ and $\ell \geqslant 2$ be integers. Assume that there is an element $\zeta \in$ $[Y, K(\mathbb{Z} / \ell, m+2)]$ such that $\zeta^{\sharp}:[K(\mathbb{Z} / \ell, m+2), K(\mathbb{Z} / \ell, m+2)] \rightarrow[Y, K(\mathbb{Z} / \ell, m+2)]$ is an isomorphism, that is,

$$
[Y, K(\mathbb{Z} / \ell, m+2)]=\{a \circ \zeta \mid a \in[K(\mathbb{Z} / \ell, m+2), K(\mathbb{Z} / \ell, m+2)]\} \cong \mathbb{Z} / \ell .
$$

(1) If $f \in \mathcal{E}(Y)$, then there exists an element $u \in \mathbb{Z} / \ell$ such that $\zeta \circ f=h_{u} \circ \zeta$ and $(u, \ell)=1$.

(2) If $f \in \mathcal{E}(Y)$ and $\gamma=h_{c} \circ \zeta$ for some $c \in \mathbb{Z} / \ell$, then we have $\gamma \circ f=h_{u} \circ \gamma$ for the element $u \in \mathbb{Z} / \ell$ in $(1)$.

(3) Let $\gamma=h_{c} \circ \zeta$ for some $c \in \mathbb{Z} / \ell$ and $\ell=w \ell^{\prime}$ and $c=w c^{\prime}$ with $\left(\ell^{\prime}, c^{\prime}\right)=1$. Let $f \in \mathcal{E}(Y)$ and $\gamma \circ f=h_{u} \circ \gamma$ for the element $u \in \mathbb{Z} / \ell$ as in (2). Assume that $\gamma \circ f=h_{t} \circ \gamma$ for another element $t \in \mathbb{Z} / \ell$. Then,

$(3-1)(t, \ell)=1$ if $\ell^{\prime}$ contains any prime divisor of $\ell$;

$(3-2)(t, \ell)=1$ does not always hold if there exists a prime $p$ such that $(p, w)=p$ and $\left(p, \ell^{\prime}\right)=1$. 
Proof. (1) Since $f \in \mathcal{E}(Y)$, the induced homomorphism

$$
f^{\sharp}:[Y, K(\mathbb{Z} / \ell, m+2)] \cong \mathbb{Z} / \ell \rightarrow[Y, K(\mathbb{Z} / \ell, m+2)] \cong \mathbb{Z} / \ell
$$

is an isomorphism. Therefore, $\zeta \circ f$ is also a generator of $[Y, K(\mathbb{Z} / \ell, m+2)] \cong \mathbb{Z} / \ell$, and hence there exists an element $u \in \mathbb{Z} / \ell$ such that $(u, \ell)=1$ and $\zeta \circ f=h_{u} \circ \zeta$.

(2) If $\gamma=h_{c} \circ \zeta$ for some $c \in \mathbb{Z} / \ell$, then we have

$$
\gamma \circ f=h_{c} \circ \zeta \circ f=h_{c} \circ h_{u} \circ \zeta=h_{u} \circ h_{c} \circ \zeta=h_{u} \circ \gamma .
$$

(3) We have the results (3-1) and (3-2) by Lemma 3.15(1) and (2), respectively.

Theorem 3.18. Let $\ell \geqslant 2$ and $\ell=w \ell^{\prime}$ and $c=w c^{\prime}$ with $\left(\ell^{\prime}, c^{\prime}\right)=1$ for some integers $w, \ell^{\prime}, c^{\prime} \geqslant 1$ such that $\ell^{\prime}$ contains any prime divisor of $\ell$. Let $m \geqslant 0$. Assume that $Y$ is a 0 -connected $C W$-complex and $\pi_{d}(Y)=0$ for $d \geqslant m+1$ in the following fibration sequence:

$$
\cdots \longrightarrow \Omega Y \stackrel{\Omega \gamma}{\longrightarrow} \Omega K(\mathbb{Z} / \ell, m+2) \stackrel{q}{\longrightarrow} X \stackrel{i}{\longrightarrow} Y \stackrel{\gamma}{\longrightarrow} K(\mathbb{Z} / \ell, m+2) .
$$

If there exists an element $\zeta \in[Y, K(\mathbb{Z} / \ell, m+2)]$ such that

$$
[Y, K(\mathbb{Z} / \ell, m+2)]=\{a \circ \zeta \mid a \in[K(\mathbb{Z} / \ell, m+2), K(\mathbb{Z} / \ell, m+2)]\} \cong \mathbb{Z} / \ell
$$

and $\gamma=c \zeta$, then $N \mathcal{E}(X)=N \mathcal{E}(Y)$.

Proof. Assume that $N \mathcal{E}(Y)=k(\leqslant m)$. Let $g: X \rightarrow X$ be a map which satisfies

$$
g_{\sharp}: \pi_{d}(X) \rightarrow \pi_{d}(X)
$$

is an isomorphism for any $d \leqslant k \leqslant m$. By the argument in Nomura [14], there exist maps $f: Y \rightarrow Y$ and $s: K(\mathbb{Z} / \ell, m+2) \rightarrow K(\mathbb{Z} / \ell, m+2)$ which make the following diagram homotopy commutative:

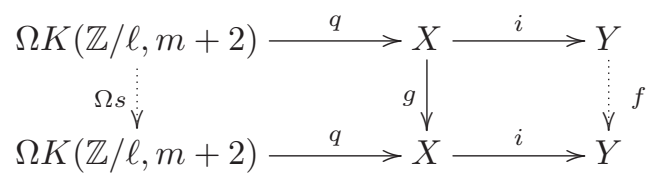

We see $f_{\sharp}: \pi_{d}(Y) \rightarrow \pi_{d}(Y)$ is an isomorphism for any $d \leqslant k$, since $i_{\sharp}: \pi_{d}(X) \rightarrow \pi_{d}(Y)$ is an isomorphism for any $d \leqslant m$ by Proposition 3.1 and $g_{\sharp}: \pi_{d}(X) \rightarrow \pi_{d}(X)$ is an isomorphism for any $d \leqslant k$. Hence $f \in \mathcal{E}(Y)$ since $N \mathcal{E}(Y)=k$.

We see $\gamma \circ f=u \circ \gamma$ for an element $u \in[K(\mathbb{Z} / \ell, m+2), K(\mathbb{Z} / \ell, m+2)]$ such that $(u, \ell)=1$ by Lemma $3.17(1)(2)$ since $f \in \mathcal{E}(Y)$ as in the following diagram:

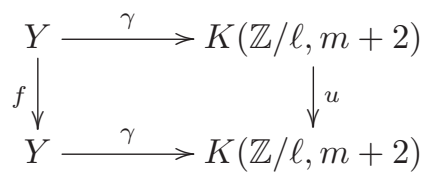

By Theorem 2.6 there exists an element $t \in[K(\mathbb{Z} / \ell, m+2), K(\mathbb{Z} / \ell, m+2)]$ and elements $a, b \in \mathcal{E}(K(\mathbb{Z} / \ell, m+2))$ such that $s=a \circ t \circ b$ in the following homotopy 
commutative diagram:

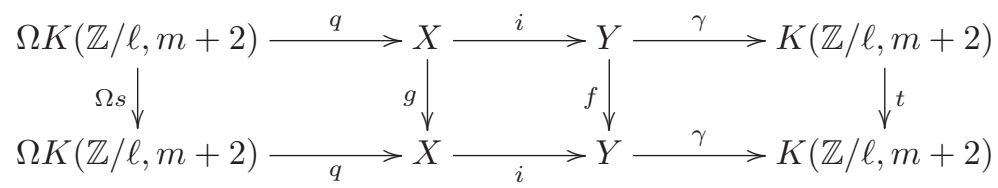

By Lemma 3.17(3) we see $(t, \ell)=1$ and hence $t$ is a homotopy equivalence. Therefore, $f \in \mathcal{E}(Y)$ and the maps $t: K(\mathbb{Z} / \ell, m+2) \rightarrow K(\mathbb{Z} / \ell, m+2), \Omega s: \Omega K(\mathbb{Z} / \ell, m+2) \rightarrow$ $\Omega K(\mathbb{Z} / \ell, m+2)$ are homotopy equivalences, and hence $g \in \mathcal{E}(X)$ by the five lemma applied to the long homotopy exact sequences. It follows that $N \mathcal{E}(X) \leqslant k=N \mathcal{E}(Y)$. Therefore, we have $N \mathcal{E}(X)=N \mathcal{E}(Y)$ by Proposition 3.4.

Example 3.19. The following are examples of Proposition 3.13 and Theorem 3.18.

(1) Let $Y=\mathbb{C} P^{\infty}$ and $H^{*}\left(\mathbb{C} P^{\infty}\right)=\mathbb{Z}[x]$, where $\operatorname{deg}(x)=2$.

Let $\overline{x^{2}}: \mathbb{C} P^{\infty} \rightarrow K(\mathbb{Z}, 4)$ be a map which represents $x^{2} \in H^{4}\left(\mathbb{C} P^{\infty}\right)$. Consider the following fibration sequence for $c \geqslant 1$ :

$$
\cdots \longrightarrow \Omega \mathbb{C} P^{\infty} \stackrel{\Omega\left(c \overline{x^{2}}\right)}{\longrightarrow} \Omega K(\mathbb{Z}, 4) \stackrel{q}{\longrightarrow} X \stackrel{i}{\longrightarrow} \mathbb{C} P^{\infty} \stackrel{c \overline{x^{2}}}{\longrightarrow} K(\mathbb{Z}, 4) .
$$

Then we have $N \mathcal{E}(X)=N \mathcal{E}\left(\mathbb{C} P^{\infty}\right)=2$ by Proposition 3.13 for $G=\mathbb{Z}$.

(2) Let $Y=\mathbb{C} P^{\infty}$ and $H^{*}\left(\mathbb{C} P^{\infty} ; \mathbb{Z} / 8\right)=\mathbb{Z} / 8[x]$, where $\operatorname{deg}(x)=2$.

Let $\overline{x^{2}}: \mathbb{C} P^{\infty} \rightarrow K(\mathbb{Z} / 8,4)$ be a map which represents $x^{2} \in H^{4}\left(\mathbb{C} P^{\infty} ; \mathbb{Z} / 8\right)$.

Consider the following fibration sequence for $c=2$ :

$$
\cdots \longrightarrow \Omega \mathbb{C} P^{\infty} \stackrel{\Omega\left(2 \overline{x^{2}}\right)}{\longrightarrow} \Omega K(\mathbb{Z} / 8,4) \stackrel{q}{\longrightarrow} X \stackrel{i}{\longrightarrow} \mathbb{C} P^{\infty} \stackrel{2 \overline{x^{2}}}{\longrightarrow} K(\mathbb{Z} / 8,4) .
$$

Then we have $N \mathcal{E}(X)=N \mathcal{E}\left(\mathbb{C} P^{\infty}\right)=2$ by Theorem 3.18 for $G=\mathbb{Z} / 8$.

\subsection{Another inequation}

Let $\pi_{*}-\operatorname{dim}(Z):=\max \left\{i \mid \pi_{i}(Z) \neq 0\right\}$ and $\operatorname{conn}(Z):=\min \left\{i \geqslant 0 \mid \pi_{i+1}(Z) \neq 0\right\}$.

Theorem 3.20. Let $\xi: F \rightarrow X \rightarrow Y$ be a fibration for connected $C W$ complexes $F$, $X$ and $Y$. If $\pi_{*}-\operatorname{dim}(Y) \leqslant \operatorname{conn}(F)$, then $N \mathcal{E}(F) \geqslant N \mathcal{E}(X)$.

Proof. Let $\operatorname{conn}(F)=m$. Assume that $N \mathcal{E}(F)=k(>m)$. Let $g: X \rightarrow X$ be a map which satisfies

$$
g_{\sharp}: \pi_{d}(X) \rightarrow \pi_{d}(X)
$$

is an isomorphism for any $d \leqslant k$. By the argument of Nomura [14], there exist maps $f: Y \rightarrow Y$ and $h: F \rightarrow F$ which make the following diagram homotopy commutative:

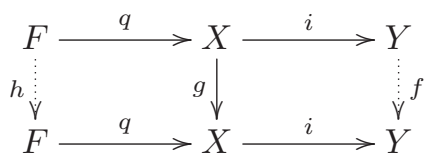

Then from the long homotopy exact sequences of $\xi$ we have the following commutative 
diagram:

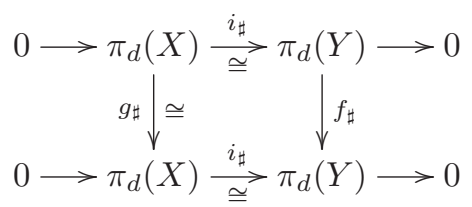

where $i_{\sharp}: \pi_{d}(X) \rightarrow \pi_{d}(Y)$ is an isomorphism for any $d \leqslant m$. Thus $f_{\sharp}$ is an isomorphism for any $d \geqslant 1$ by the assumption $\pi_{*}$ - $\operatorname{dim}(Y) \leqslant \operatorname{conn}(F)$, and hence $f \in \mathcal{E}(Y)$. Moreover, we have the following commutative diagram:

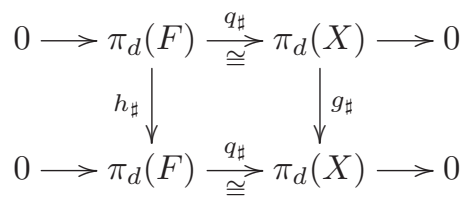

where $q_{\sharp}: \pi_{d}(F) \rightarrow \pi_{d}(X)$ is an isomorphism for any $d>m$. From our assumption, $h_{\sharp}$ is an isomorphism for any $d \leqslant k$. Thus $h_{\sharp}$ is an isomorphism for any $d \geqslant 1$ since $N \mathcal{E}(F)=k$. By applying the five lemma for the long homotopy exact sequences of $\xi$, we see that $g_{\sharp}$ is an isomorphism for any $d$. Hence $g \in \mathcal{E}(X)$.

\section{Rational results}

In this section, we assume that a space is a nilpotent CW complex of finite type. Let $X_{0}$ be the rationalization of a space $X[\mathbf{1 2}]$. Then $\pi_{*}\left(X_{0}\right)=\pi_{*}(X) \otimes \mathbb{Q}$ and $H_{*}\left(X_{0} ; \mathbb{Z}\right)=H_{*}(X ; \mathbb{Q})$. We assume familiarity with rational homotopy theory as in the text $[4]$.

\subsection{Sullivan models of fibrations}

Let $M(X)=(\Lambda V, d)$ be the Sullivan minimal model of a nilpotent space $X[\mathbf{1 6}]$. It is a free commutative differential graded algebra over $\mathbb{Q}(D G A)$ with a $\mathbb{Q}$-graded vector space $V=\bigoplus_{i \geqslant 1} V^{i}$ where $\operatorname{dim} V^{i}<\infty$ and a decomposable differential, namely $d\left(V^{i}\right) \subset\left(\Lambda^{+} V \cdot \Lambda^{+} V\right)^{i+1}$ and $d \circ d=0$. Here $\Lambda^{+} V$ is the ideal of $\Lambda V$ generated by elements of positive degree. The degree of a homogeneous element $x$ of a graded algebra is denoted by $|x|$. Then $x y=(-1)^{|x||y|} y x$ and $d(x y)=d(x) y+(-1)^{|x|} x d(y)$. Note that $M(X)$ determines the rational homotopy type of $X$. In particular, $V^{*} \cong$ $\operatorname{Hom}\left(\pi_{*}(X), \mathbb{Q}\right)$ and $H^{*}(\Lambda V, d) \cong H^{*}(X ; \mathbb{Q})$. Here the second is an isomorphism as graded algebras. Furthermore, for $d=d_{2}+d_{3}+\cdots$ with $d_{i} V \subset \Lambda^{i} V$ (the vector space generated by the products $v_{1} \cdots v_{k} \cdots v_{i}$ with $\left.v_{k} \in V\right)$ [17, p. 9], the quadratic part $d_{2}: V \rightarrow \Lambda^{2} V$ is the dual of the rational Whitehead product

$$
[,]: \pi_{p}(X)_{\mathbb{Q}} \otimes \pi_{q}(X)_{\mathbb{Q}} \rightarrow \pi_{p+q-1}(X)_{\mathbb{Q}} .
$$

Here $\pi_{n}(X)_{\mathbb{Q}}:=\pi_{n}(X) \otimes \mathbb{Q}$ (see $[\mathbf{1 7}$, p. 26] for detail).

Now we recall "DGA-homotopy" in Chapter $X$ of [8]: In general, two maps $f: M(Y) \rightarrow M(X)$ and $g: M(Y) \rightarrow M(X)$ are DGA-homotopic (denote as $f \sim g$ ) if there is a DGA-map $H: M(Y) \rightarrow M(X) \otimes \Lambda(t, d t)$ such that $\left.H\right|_{t=0, d t=0}=f$ and 
$\left.H\right|_{t=1, d t=0}=g$. Here $|t|=0$ and $|d t|=1$ with $d(t)=d t, d(d t)=0$. Then we have

$$
\left[X_{0}, Y_{0}\right] \cong[M(Y), M(X)]
$$

as homotopy sets. Let Aut $M$ be the group of DGA-automorphisms of a DGA $M$. For a nilpotent space $X$ and the model $M(X)$, there is a group isomorphism

$$
\mathcal{E}\left(X_{0}\right) \cong \mathcal{E}(M(X)):=\operatorname{Aut} M(X) / \sim,
$$

which is the group of self-DGA-homotopy equivalence classes of $M(X)$. Thus we have the rational self-closeness number of $X$ as $N \mathcal{E}\left(X_{0}\right)=N \mathcal{E}(M(X))$.

A map $f: X \rightarrow Y$ has a minimal model which is a DGA-map $M(f): M(Y) \rightarrow$ $M(X)$. It is induced by a relative or Koszul-Sullivan (KS-)model

$$
i: M(Y)=\left(\Lambda W, d_{Y}\right) \rightarrow(\Lambda W \otimes \Lambda V, D) .
$$

Here, $\left.D\right|_{W}=d_{Y}$ and $(\Lambda V, \bar{D})=(\Lambda V, d)$, where $\bar{D}$ is induced by $D$, is the minimal model of the homotopy fibre of $f$ and there is a quasi-isomorphism $\rho_{X}: M(X) \stackrel{\sim}{\rightarrow}$ $(\Lambda W \otimes \Lambda V, D)$ such that $\rho_{X} \circ M(f) \simeq i$. We refer the reader to [9] for the rational fibration and the Sullivan model.

Example 4.1. For example, we consider a fibration $S^{m+n-1} \rightarrow X \rightarrow Y=S^{m} \times S^{n}$ with non-zero Euler class for odd-integers $m, n \geqslant 3$ with $m<n$ : The rational model of the fibration is given by

$$
M(Y)=(\Lambda(x, y), 0) \rightarrow(\Lambda(x, y, z), D) \rightarrow(\Lambda z, 0)=M\left(S^{m+n-1}\right)
$$

with $|x|=m,|y|=n,|z|=m+n-1, D x=D y=0$ and $D z=x y$. Since $D f(z)=$ $f(x) f(y)$ for any $f \in[(\Lambda(x, y, z), D),(\Lambda(x, y, z), D)]$, we have $N \mathcal{E}\left(X_{0}\right)=\max \{m, n\}$. Moreover, we may put $f(x)=a x, f(y)=b y$ and $f(z)=a b z$ for $a, b \in \mathbb{Q}$ for reason of the degrees, and hence we have

$$
[(\Lambda(x, y, z), D),(\Lambda(x, y, z), D)] \cong \mathbb{Q} \times \mathbb{Q}
$$

and $\mathcal{E}(\Lambda(x, y, z), D) \cong \mathbb{Q}^{*} \times \mathbb{Q}^{*}$ for $\mathbb{Q}^{*}=\mathbb{Q}-\{0\}$.

Example 4.2. (1) There is a rationalized fibration

$$
F=S_{0}^{2 n-1} \rightarrow X=\left(S^{n} \times S^{m n-1}\right)_{0} \rightarrow Y,
$$

where $Y$ is a rational space with $H^{*}(Y ; \mathbb{Q})=\mathbb{Q}[x] /\left(x^{m}\right)$ with $|x|=n$ even. It is given by the KS-model

$$
\left(\Lambda(x, y), d_{Y}\right) \rightarrow(\Lambda(x, y, z), D) \rightarrow(\Lambda z, 0)
$$

with $|y|=m n-1,|z|=2 n-1, d_{Y}(y)=x^{m}, D z=x^{2}$. Then $N \mathcal{E}(F)=2 n-1$, $N \mathcal{E}(Y)=n$ and $N \mathcal{E}(X)=m n-1$. Note that $(\Lambda(x, y, z), D) \cong M\left(S^{n} \times S^{m n-1}\right)$ by a suitable change of basis. Thus $N \mathcal{E}(X)$ can be arbitrary large for $N \mathcal{E}(F)$ and $N \mathcal{E}(Y)$.

(2) Let $H^{*}(C ; \mathbb{Q})=\mathbb{Q}[y] /\left(y^{3}\right)$ with $|y|=6$ and $F=\left(\mathbb{C} P^{7} \times \mathbb{H} P^{2}\right)_{0} \rightarrow X \rightarrow Y=$ $\left(C \times S^{10}\right)_{0}$ be a rationalized fibration given by the KS-model

$$
\left(\Lambda\left(y, z, u_{1}, u_{2}\right), d_{Y}\right) \rightarrow\left(\Lambda\left(y, z, u_{1}, u_{2}, x, w, v_{1}, v_{2}\right), D\right) \rightarrow\left(\Lambda\left(x, w, v_{1}, v_{2}\right), \bar{D}\right)
$$

with $|z|=10,\left|u_{1}\right|=17,\left|u_{2}\right|=19,|x|=2,|w|=4,\left|v_{1}\right|=15,\left|v_{2}\right|=11, \bar{D}\left(v_{1}\right)=x^{8}$, $\bar{D}\left(v_{2}\right)=w^{3}, d_{Y}\left(u_{1}\right)=y^{3}, d_{Y}\left(u_{2}\right)=z^{2}$,

$$
D\left(v_{1}\right)=x^{8}+y z \text { and } D\left(v_{2}\right)=w^{3}+y^{2} .
$$

Then we have that $N \mathcal{E}(X)<N \mathcal{E}(F)<N \mathcal{E}(Y)$ since $N \mathcal{E}(F)=4, N \mathcal{E}(Y)=10$ and 
$N \mathcal{E}(X)=2$. Indeed, for $f \in[X, X]$, we have $f(x)=a x, f(w)=b w+h_{1}(x), f(y)=$ $c y+h_{2}(x, w), f(z)=e z+h_{3}(x, y, w)$ for $a, b, c, e \in \mathbb{Q}$. Here $h_{i}$ are polynomials. Then we have $a^{8}=c e$ and $b^{3}=c^{2}$. Therefore if $a \neq 0$, then we have $b \neq 0, c \neq 0$ and $e \neq 0$. Thus if $\pi_{2}(f)$ is an isomorphism, then we have $f \in \mathcal{E}(X)$. Hence $N \mathcal{E}(X)=2$.

Example 4.3. We consider fibrations $F \rightarrow X \rightarrow Y$ of rational spaces in Proposition 3.4.

(1) In Proposition 3.4, we need the condition that $\pi_{d}(Y)=0$ for $d \geqslant m+1$. For example, let $X=S_{0}^{3}, Y=\left(S^{3} \times S^{5}\right)_{0}$ and $m=3$. Then there exists a fibration

$$
K(\mathbb{Q}, 4) \rightarrow S_{0}^{3} \rightarrow\left(S^{3} \times S^{5}\right)_{0} \stackrel{p_{2}}{\longrightarrow} K(\mathbb{Q}, 5),
$$

where $p_{2}$ is the projection to the second factor. Then $N \mathcal{E}(X)=3<5=N \mathcal{E}(Y)$.

(2) In Proposition 3.4, we need the condition that $F=K(G, m+1)$ : Let $F=\mathbb{C} P_{0}^{3}$ and $Y=\left(S^{3} \times S^{5}\right)_{0}$. Let $M(F)=\left(\Lambda(u, v), d_{X}\right)$ with $|u|=2,|v|=7, d_{X}(u)=$ $0, d_{X}(v)=u^{4}$ and $M(Y)=(\Lambda(x, y), 0)$ with $|x|=3,|y|=5$. Then there is a fibration

$$
\mathbb{C} P_{0}^{3} \rightarrow X \rightarrow\left(S^{3} \times S^{5}\right)_{0}
$$

given by the KS-model

$$
(\Lambda(x, y), 0) \rightarrow(\Lambda(x, y, u, v), D) \rightarrow\left(\Lambda(u, v), d_{X}\right),
$$

where $D(x)=D(y)=D(u)=0$ and $D(v)=u^{4}+x y$. Notice that $N \mathcal{E}(X)=2$ : Indeed, if $f(u)=a u$ with $a \neq 0 \in \mathbb{Q}$ for $f \in[(\Lambda(x, y, u, v), D),(\Lambda(x, y, u, v), D)]$, then we have $f(x)=b x, f(y)=c y+c^{\prime} x u$ for some $b, c, c^{\prime} \in \mathbb{Q}$. Then $a^{4}=b c$ from the relation $f(D(v))=D(f(v))$. Thus $N \mathcal{E}(X)=2<5=N \mathcal{E}(Y)$.

On the other hand, we have a fibration

$$
K(\mathbb{Q}, 7) \rightarrow X^{\prime} \rightarrow\left(S^{3} \times S^{5}\right)_{0}
$$

given by the KS-model

$$
(\Lambda(x, y), 0) \rightarrow(\Lambda(x, y, v), D) \rightarrow(\Lambda(v), 0),
$$

where $|v|=7$ and $D(v)=x y$. Then $N \mathcal{E}\left(X^{\prime}\right)=5=N \mathcal{E}(Y)$.

(3) In Proposition 3.4, we need the condition that

$$
[Y, K(G, m+2)]=\{a \circ \zeta \mid a \in[K(G, m+2), K(G, m+2)]\} .
$$

See, for example, Theorem 4.10(i) in Section 4.3.

Example 4.4. We consider fibrations $F \rightarrow X \rightarrow Y$ of rational spaces in Theorem 3.9.

(1) In Theorem 3.9, we need the condition that $\pi_{d}(Y)=0$ for $d \geqslant m+1$. For example, let $X=\left(S^{2} \times S^{5}\right)_{0}$ and $Y=\mathbb{C} P_{0}^{2}$. There exists a fibration $K(\mathbb{Q}, 3) \stackrel{q}{\rightarrow}$ $\left(S^{2} \times S^{5}\right)_{0} \stackrel{i}{\rightarrow} Y=\mathbb{C} P_{0}^{2}$ given by the KS-model

$$
\left(\Lambda(u, v), d_{Y}\right) \rightarrow(\Lambda(u, v, x), D) \rightarrow(\Lambda(x), 0)
$$

with $|u|=2,|v|=5,|x|=3, d_{Y} u=0, d_{Y} v=u^{3}$ and $D x=u^{2}$. It follows then that $N \mathcal{E}(X)=5>2=N \mathcal{E}(Y)$. In this case, $m=2$ but $\pi_{5}(Y) \neq 0$.

(2) In Theorem 3.9, we need the condition that $F=K(\mathbb{Q}, m+1)$ : 
Let $X=S_{0}^{2} \times K(\mathbb{Q}, 5)$ and $Y=K(\mathbb{Q}, 2)$. There exists a fibration

$$
F=K(\mathbb{Q}, 3) \times K(\mathbb{Q}, 5) \stackrel{q}{\longrightarrow} S_{0}^{2} \times K(\mathbb{Q}, 5) \stackrel{i}{\longrightarrow} K(\mathbb{Q}, 2)
$$

given by the KS-model

$$
(\Lambda u, 0) \rightarrow(\Lambda(u, x, y), D) \rightarrow(\Lambda(x, y), 0)
$$

with $|u|=2,|x|=3,|y|=5, D x=u^{2}$ and $D y=u^{3}$. It follows that

$$
N \mathcal{E}(X)=5>2=N \mathcal{E}(Y) .
$$

(3) In Theorem 3.9, we need the condition that $\gamma^{\sharp}$ is a monomorphism. We note that Remark 3.5 is an example where $\gamma^{\sharp}=0$.

Theorem 4.5. Suppose that a Lie group $G$ acts on a space $X$. Let $\left(n_{1}, \ldots, n_{l}\right)$ with $n_{1} \leqslant \cdots \leqslant n_{l}$ be the rational type of $G$ and $n_{l}<\operatorname{conn}(X)$. Then

$$
N \mathcal{E}\left(X_{0}\right) \geqslant N \mathcal{E}\left(\left(E G \times_{G} X\right)_{0}\right) .
$$

Proof. There is a Borel fibration $X \rightarrow E G \times_{G} X \rightarrow B G$ (p. 69 of [1]). Then the result follows by Theorem 3.20.

Example 4.6. Let $X=S^{3} \times S^{5} \times S^{7} \times S^{9} \times S^{13}$. Then

$$
M(X)=\left(\Lambda\left(v_{1}, v_{2}, v_{3}, v_{4}, v_{5}\right), 0\right)
$$

with $\left|v_{1}\right|=3,\left|v_{2}\right|=5,\left|v_{3}\right|=7,\left|v_{4}\right|=9,\left|v_{5}\right|=13$. Note that $\left[X_{0}, X_{0}\right] \cong \mathbb{Q}^{\times 5}$ and $\mathcal{E}\left(X_{0}\right) \cong\left(\mathbb{Q}^{*}\right)^{\times 5}$ with $\mathbb{Q}^{*}=\mathbb{Q}-\{0\}$ by $f\left(v_{i}\right)=a_{i} v_{i}\left(a_{i} \in \mathbb{Q}\right)$ for $i=1, \ldots, 5$. According to $[\mathbf{1 0}]$ there are Borel fibrations of certain free $S^{1}$-actions

$$
X \rightarrow E S^{1} \times S^{1} X \rightarrow B S^{1}
$$

given by the KS-models

$$
(\mathbb{Q}[t], 0) \rightarrow\left(\mathbb{Q}[t] \otimes \Lambda\left(v_{1}, v_{2}, v_{3}, v_{4}, v_{5}\right), D\right) \rightarrow\left(\Lambda\left(v_{1}, v_{2}, v_{3}, v_{4}, v_{5}\right), 0\right)
$$

with $|t|=2$ and $\operatorname{dim} H^{*}\left(\mathbb{Q}[t] \otimes \Lambda\left(v_{1}, v_{2}, v_{3}, v_{4}, v_{5}\right), D\right)<\infty$. (In general $E G \times_{G} X \simeq$ $X / G$ when the $G$-action is free.) Note that the DGA-homotopy set

$$
\left[\left(X / S^{1}\right)_{0},\left(X / S^{1}\right)_{0}\right]=\left[\left(\Lambda\left(t, v_{1}, v_{2}, v_{3}, v_{4}, v_{5}\right), D\right),\left(\Lambda\left(t, v_{1}, v_{2}, v_{3}, v_{4}, v_{5}\right), D\right)\right]
$$

is a set given by the elements $f$ with $a_{i}, b_{i}, b_{i}^{\prime}, \ldots \in \mathbb{Q}$ :

$$
\begin{aligned}
f(t) & =a_{0} t, \quad f\left(v_{1}\right)=a_{1} v_{1}, \quad f\left(v_{2}\right)=a_{2} v_{2}+b_{2} v_{1} t, \quad f\left(v_{3}\right)=a_{3} v_{3}+b_{3} v_{2} t+b_{3}^{\prime} v_{1} t^{2}, \\
f\left(v_{4}\right) & =a_{4} v_{4}+b_{4} v_{3} t+b_{4}^{\prime} v_{2} t^{2}+b_{4}^{\prime \prime} v_{1} t^{3} \quad \text { and } \\
f\left(v_{5}\right) & =a_{5} v_{5}+b_{5} v_{4} t^{2}+b_{5}^{\prime} v_{3} t^{3}+b_{5}^{\prime \prime} v_{2} t^{4}+b_{5}^{\prime \prime \prime} v_{1} t^{5} .
\end{aligned}
$$

Then $N \mathcal{E}\left(\left(X / S^{1}\right)_{0}\right) \leqslant 13$ by Theorem 4.5. In particular,

(1) If $D v_{5}=v_{1} v_{4} t+v_{2} v_{3} t+t^{7}, D v_{1}=D v_{2}=D v_{3}=D v_{4}=0$, then $N \mathcal{E}\left(X / S^{1}\right)=2$.

(2) If $D v_{5}=v_{2} v_{3} t+t^{7}, D v_{4}=v_{1} v_{2} t, D v_{1}=D v_{2}=D v_{3}=0$, then $N \mathcal{E}\left(X / S^{1}\right)=3$.

(3) If $D v_{5}=v_{1} v_{4} t, D v_{4}=v_{1} v_{2} t, D v_{3}=t^{4}, D v_{1}=D v_{2}=0$, then $N \mathcal{E}\left(X / S^{1}\right)=5$.

(4) If $D v_{5}=v_{1} v_{4} t+t^{7}, D v_{1}=D v_{2}=D v_{3}=D v_{4}=0$, then $N \mathcal{E}\left(X / S^{1}\right)=7$.

(5) If $D v_{5}=v_{2} v_{3} t+t^{7}, D v_{1}=D v_{2}=D v_{3}=D v_{4}=0$, then $N \mathcal{E}\left(X / S^{1}\right)=9$. 
(6) If $D v_{4}=t^{5}, D v_{1}=D v_{2}=D v_{3}=D v_{5}=0$, then $N \mathcal{E}\left(X / S^{1}\right)=13$.

Indeed, for example, in (1), if $a_{0} \neq 0$, that is, $\pi_{2}(f)$ is an isomorphism, then $a_{5}=a_{0}^{7}$, $a_{1} a_{4}=a_{2} a_{3}=a_{0}^{6}$ since $f$ is a DGA-map. Thus we have $f \in \mathcal{E}\left(\Lambda\left(t, v_{1}, v_{2}, v_{3}, v_{4}, v_{5}\right), D\right)$. Hence $N \mathcal{E}\left(X / S^{1}\right)=2$.

\subsection{A dual result of the case of cofibrations}

In this subsection, we give an Eckmann-Hilton dual result of cofibrations studied in Theorems 3 and 4 of [15]. The following proposition is proved by making use of the Sullivan model for the case where $G=\mathbb{Z}$ and $[Y, K(\mathbb{Z}, m+2)]$ is a free group, which is a special case of Theorem 2.6.

Proposition 4.7. Let $m \geqslant 2$ and $K(\mathbb{Z}, m+1) \stackrel{q}{\rightarrow} X \stackrel{i}{\rightarrow} Y \stackrel{\gamma}{\rightarrow} K(\mathbb{Z}, m+2)$ be a fibration sequence such that $Y$ is 1 -connected and $\pi_{k}(Y)=0$ for $k \geqslant m+1$. Assume that there exist maps $f: Y \rightarrow Y, g: X \rightarrow X$ and $s \mathbf{1}_{\Omega K}: K(\mathbb{Z}, m+1) \rightarrow K(\mathbb{Z}, m+1)$ for some $s \in \mathbb{Z}$, which make the following diagram homotopy commutative:

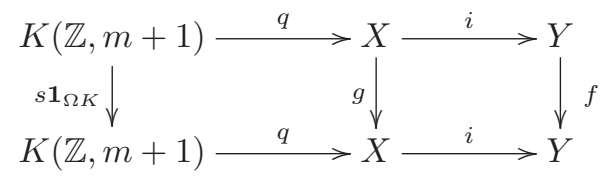

If $H^{m+2}(Y ; \mathbb{Z})$ is torsion-free, then there is the homotopy commutative diagram:

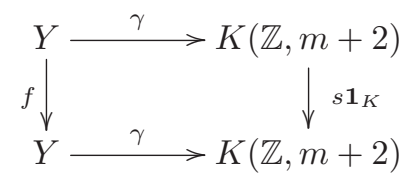

Proof. The Sullivan model of the fibration $K(\mathbb{Z}, m+1) \stackrel{q}{\rightarrow} X \stackrel{i}{\rightarrow} Y$ is given by

$M(Y)=\left(\Lambda V_{Y}, d_{Y}\right) \stackrel{i^{*}}{\longrightarrow} M(X)=\left(\Lambda \bar{u} \otimes \Lambda V_{Y}, d_{X}\right) \stackrel{q^{*}}{\longrightarrow} M(K(\mathbb{Z}, m+1))=(\Lambda \bar{u}, 0)$,

where $|\bar{u}|=m+1, d_{X}(\bar{u}) \in \Lambda V_{Y}$ and $\left.d_{X}\right|_{V_{Y}}=d_{Y}$. Here $V_{Y}^{1}=0$ and $V_{Y}^{k}=0$ for $k \geqslant$ $m+1$.

Then the model of the diagram (1) is given by

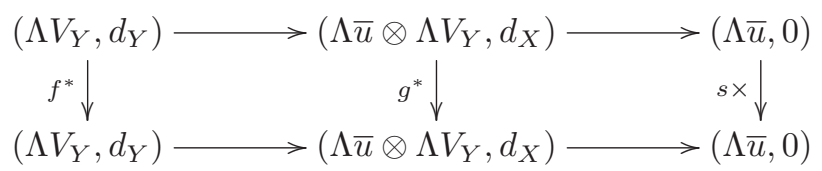
by

On the other hand, the Sullivan model of fibration $X \stackrel{i}{\rightarrow} Y \stackrel{\gamma}{\rightarrow} K(\mathbb{Z}, m+2)$ is given

$$
M(K(\mathbb{Z}, m+2))=(\Lambda u, 0) \stackrel{\gamma^{*}}{\rightarrow}\left(\Lambda u \otimes \Lambda \bar{u} \otimes \Lambda V_{Y}, D\right) \stackrel{i^{*}}{\rightarrow} M(X)=\left(\Lambda \bar{u} \otimes \Lambda V_{Y}, d_{X}\right),
$$

where $|u|=m+2, D(u)=0, D(\bar{u})=u+d_{X}(\bar{u})\left(\right.$ from $\left.V_{Y}^{\geqslant m+1}=0\right)$ and $\left.D\right|_{V_{Y}}=d_{Y}$. 
Thus the Sullivan model of fibration sequence:

$$
K(\mathbb{Z}, m+1) \stackrel{q}{\longrightarrow} X \stackrel{i}{\longrightarrow} Y \stackrel{\gamma}{\longrightarrow} K(\mathbb{Z}, m+2)
$$

is given as

$$
(\Lambda u, 0) \stackrel{\gamma^{*}}{\longrightarrow}\left(\Lambda u \otimes \Lambda \bar{u} \otimes \Lambda V_{Y}, D\right) \stackrel{i^{*}}{\longrightarrow} M(X)=\left(\Lambda \bar{u} \otimes \Lambda V_{Y}, d_{X}\right) \stackrel{q^{*}}{\longrightarrow}(\Lambda \bar{u}, 0) .
$$

Note that the inclusion $\rho: M(Y)=\left(\Lambda V_{Y}, d_{Y}\right) \rightarrow\left(\Lambda u \otimes \Lambda \bar{u} \otimes \Lambda V_{Y}, D\right)$ is a quasiisomorphism and the right hand model is not minimal.

Then the model (2) is extended as the homotopy commutative diagram:

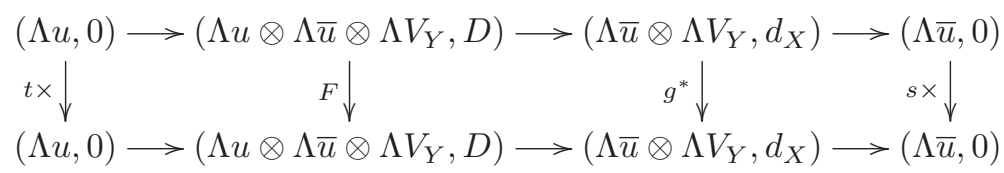

where $t \in \mathbb{Q}, F(u)=t u, F(\bar{u})=s \bar{u}$ and $\left.F\right|_{\Lambda V_{Y}}=f^{*}$. Here we remark that $H^{*}(F)=$ $H^{*}\left(f^{*}\right): H^{*}(Y ; \mathbb{Q}) \rightarrow H^{*}(Y ; \mathbb{Q})$.

Since $D \circ f^{*}=f^{*} \circ D$ we have

$$
s u+s d_{X}(\bar{u})=D(s \bar{u})=D(F(\bar{u}))=F(D(\bar{u}))=F\left(u+d_{X}(\bar{u})\right)=t u+s d_{X}(\bar{u}) .
$$

It follows that $s u=t u$ and hence $t=s$.

Thus we get the homotopy commutative diagram:

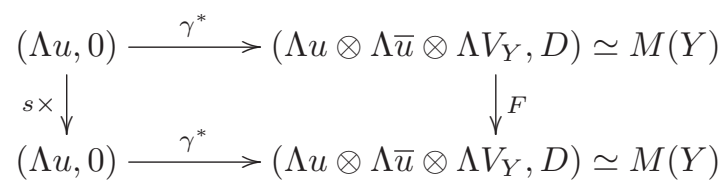

that is, we obtain the homotopy commutative diagram:

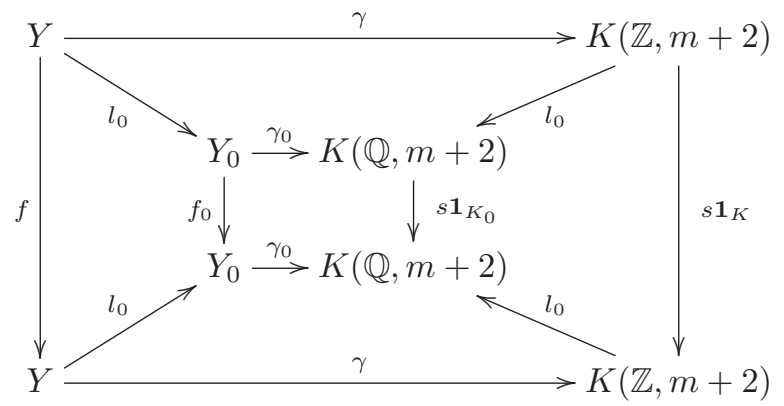

where $l_{0}$ is the rationalization map and $f_{0}$ and $\gamma_{0}$ are the rationalizations of $f$ and $\gamma$, respectively. We note that $s$ is an integer. Since $H^{m+2}(Y ; \mathbb{Z})$ is torsion-free, we see $\left(l_{0}\right)_{*}: H^{m+2}(Y ; \mathbb{Z}) \rightarrow H^{m+2}(Y ; \mathbb{Q})$ is injective, and hence we have the result.

For the case $G=\mathbb{Z}$ and $H^{m+2}(Y ; Z)$ is torsion free, we have the following result without the additional conditions in Theorem 3.9. 
Theorem 4.8. Let $m \geqslant 2$ and $K(\mathbb{Z}, m+1) \stackrel{q}{\rightarrow} X \stackrel{i}{\rightarrow} Y \stackrel{\gamma}{\rightarrow} K(\mathbb{Z}, m+2)$ be a fibration sequence such that $Y$ is 1 -connected and $\pi_{t}(Y)=0$ for $t \geqslant m+1$. If $H^{m+2}(Y ; \mathbb{Z})$ is torsion-free and $\gamma \neq 0$, then

$$
N \mathcal{E}(X) \leqslant N \mathcal{E}(Y)(\leqslant m) .
$$

Proof. Assume that $N \mathcal{E}(Y)=k(\leqslant m)$. Let $g: X \rightarrow X$ be a map which satisfies

$$
g_{\sharp}: \pi_{t}(X) \rightarrow \pi_{t}(X)
$$

is an isomorphism for any $t \leqslant k$. By a result of Nomura [14], there exist maps $f: Y \rightarrow Y$ and $s \mathbf{1}_{\Omega K}: K(\mathbb{Z}, m+1) \rightarrow K(\mathbb{Z}, m+1)$ which make the following diagram homotopy commutative:

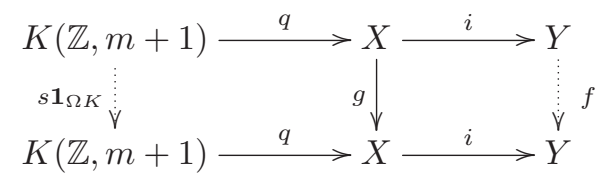

We see $f_{\sharp}: \pi_{t}(Y) \rightarrow \pi_{t}(Y)$ is an isomorphism for any $t \leqslant k$, since $i_{\sharp}: \pi_{t}(X) \rightarrow \pi_{t}(Y)$ is an isomorphism for any $t \leqslant m$ and $g_{\sharp}: \pi_{t}(X) \rightarrow \pi_{t}(X)$ is an isomorphism for any $t \leqslant k$. Hence $f \in \mathcal{E}(Y)$ since $N \mathcal{E}(Y)=k$.

By Proposition 4.7, there exists the homotopy commutative diagram:

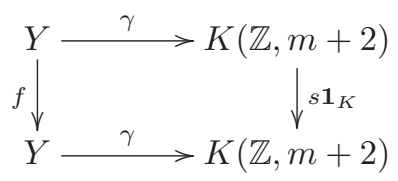

Notice that $s= \pm 1$ since

$$
f^{*}(\gamma)=\gamma \circ f=s \mathbf{1}_{K} \circ \gamma=s \gamma(\gamma \neq 0)
$$

for $f^{*}: H^{*}(Y ; \mathbb{Z}) \stackrel{\cong}{\rightarrow} H^{*}(Y ; \mathbb{Z})$.

Thus, since $s \mathbf{1}_{\Omega K} \in \mathcal{E}(K(\mathbb{Z}, m+1))$ and $f \in \mathcal{E}(Y)$, we have $g \in \mathcal{E}(X)$ by the five lemma for the long homotopy exact sequences of $(3)$. Hence $N \mathcal{E}(X) \leqslant k=N \mathcal{E}(Y)$.

By Proposition 3.4 and Theorem 4.8, we obtain the following result.

Corollary 4.9. Assume that $X \stackrel{i}{\rightarrow} Y \stackrel{\gamma}{\rightarrow} K(\mathbb{Z}, m+2)$ is a non-trivial fibration. If $H^{m+2}(Y ; \mathbb{Z}) \cong \mathbb{Z}\{\gamma\}$, then $N \mathcal{E}(X)=N \mathcal{E}(Y)$.

\subsection{A result of dichotomy}

A space $X$ is said to be formal if there is a quasi-isomorphism $M(X) \rightarrow\left(H^{*}(X\right.$; $\mathbb{Q}), 0)[3]$. For example, the $n$-sphere $S^{n}$ is formal. An $F_{0}$-space is formal [17, II.7.(8)], where a space $X$ is called an $F_{0}$-space if $H^{*}(X ; \mathbb{Q}) \cong \mathbb{Q}\left[x_{1}, \ldots, x_{n}\right] /\left(f_{1}, \ldots, f_{n}\right)$ in which $f_{1}, \ldots, f_{n}$ is a regular sequence (p. 437 of $\left.[4]\right)$. Moreover, the one-point union of formal spaces is formal [11, Lemma 1.6]. When $X$ is formal, we have $\mathcal{E}(M(X))=$ $\mathcal{E}\left(H^{*}(X ; \mathbb{Q}), 0\right)$ and $N \mathcal{E}(M(X))=N \mathcal{E}\left(H^{*}(X ; \mathbb{Q}), 0\right)$.

We obtain the following result (see [15, Theorem 16]). 
Theorem 4.10. Let $H^{*}\left(Y_{1} ; \mathbb{Q}\right) \cong \mathbb{Q}[x]$ and $H^{*}\left(Y_{2} ; \mathbb{Q}\right) \cong \mathbb{Q}[y]$ for even integers $|x|=$ $a<|y|=b<2 a$. Let $Y:=Y_{1} \vee Y_{2}$ and $n=a l=b m$ for some $l>m>1$. Then the following two cases occur for the homotopy fibre $X$ of a map $\eta: Y \rightarrow K(\mathbb{Q}, n)$ with $\eta \nsim *:$

Case 1. The following are equivalent:

(i) $N \mathcal{E}\left(X_{0}\right)=a<b=N \mathcal{E}\left(Y_{0}\right)$.

(ii) There is a proper inclusion $\mathcal{E}\left(X_{0}\right) \subsetneq \mathcal{E}\left(Y_{0}\right)$.

(iii) $X$ is an $F_{0}$-space, especially formal.

(iv) $\operatorname{dim} H^{*}(X ; \mathbb{Q})=l+m$.

Case 2. In the other case, the following are equivalent:

(i) $)^{\prime} N \mathcal{E}\left(X_{0}\right)=b=N \mathcal{E}\left(Y_{0}\right)$.

(ii) $)^{\prime} \mathcal{E}\left(X_{0}\right)=\mathcal{E}\left(Y_{0}\right)$.

(iii) $^{\prime} X$ is non-formal.

(iv) $)^{\prime} \operatorname{dim} H^{*}(X ; \mathbb{Q})=\infty$.

Proof. Let the KS-model of $\eta$ be given by

$$
g:(\mathbb{Q}[u], 0)=M(K(\mathbb{Z}, n)) \rightarrow(\Lambda(x, y, z), d)=M(Y),
$$

where $|u|=n,|x|=a,|y|=b,|z|=a+b-1, d x=d y=0, d z=x y$ and

$$
g(u)=c x^{l}+c^{\prime} y^{m}
$$

for $c, c^{\prime} \in \mathbb{Q}$. The KS-model of the associated fibration $\eta^{\prime}: K(\mathbb{Z}, n-1) \rightarrow X \rightarrow Y$ is given by

$$
(\Lambda(x, y, z), d) \rightarrow\left(\Lambda\left(x, y, z, z^{\prime}\right), D\right) \rightarrow\left(\Lambda\left(z^{\prime}\right), 0\right),
$$

where $\left|z^{\prime}\right|=n-1$ and

$$
D z=x y, D z^{\prime}=c x^{l}+c^{\prime} y^{m} .
$$

Then we have the following two cases:

Case 1. $c c^{\prime} \neq 0$ : Then we have (i)-(iv) as follows.

(i) From the assumption, we have

$$
\left[Y_{0}, Y_{0}\right]=[(\Lambda(x, y, z), d),(\Lambda(x, y, z), d)] \cong\{(\lambda, \mu) \in \mathbb{Q} \times \mathbb{Q}\} \cong \mathbb{Q} \times \mathbb{Q},
$$

where $f(x)=\lambda x, f(y)=\mu y$ and $f(z)=\lambda \mu z$ for $f \in[(\Lambda(x, y, z), d),(\Lambda(x, y, z), d)]$. Then even if $\lambda \neq 0$, it is possible that $\mu=0$. Thus $N \mathcal{E}\left(Y_{0}\right)=b$. On the other hand,

$$
\left[X_{0}, X_{0}\right]=\left[\left(\Lambda\left(x, y, z, z^{\prime}\right), D\right),\left(\Lambda\left(x, y, z, z^{\prime}\right), D\right)\right] \cong\left\{(\lambda, \mu) \in \mathbb{Q} \times \mathbb{Q} \mid \lambda^{l}=\mu^{m}\right\},
$$

where for $f \in\left[\left(\Lambda\left(x, y, z, z^{\prime}\right), D\right),\left(\Lambda\left(x, y, z, z^{\prime}\right), D\right)\right]$ we write $f(x)=\lambda x, f(y)=\mu y$, $f(z)=\lambda \mu z$ and $f\left(z^{\prime}\right)=\lambda^{l} z^{\prime}=\mu^{m} z^{\prime}$. Hence $\lambda \neq 0$ induces $\mu \neq 0$. Thus $N \mathcal{E}\left(X_{0}\right)=a$.

(ii) By an argument similar to (i), we have

$$
\begin{aligned}
\mathcal{E}\left(Y_{0}\right) & =\mathcal{E}(\Lambda(x, y, z), d) \cong\left\{(\lambda, \mu) \in \mathbb{Q}^{*} \times \mathbb{Q}^{*}\right\} \cong \mathbb{Q}^{*} \times \mathbb{Q}^{*}, \\
\mathcal{E}\left(X_{0}\right) & =\mathcal{E}\left(\Lambda\left(x, y, z, z^{\prime}\right), D\right) \cong\left\{(\lambda, \mu) \in \mathbb{Q}^{*} \times \mathbb{Q}^{*} \mid \lambda^{l}=\mu^{m}\right\} .
\end{aligned}
$$

Thus we have $\mathcal{E}\left(X_{0}\right) \subsetneq \mathcal{E}\left(Y_{0}\right)$.

(iii) It follows from $H^{*}(X ; \mathbb{Q}) \cong \mathbb{Q}[x, y] /\left(x y, c x^{l}+c^{\prime} y^{m}\right)$. 
(iv) Its basis is $\left\{1, x, \ldots, x^{l}\left(=y^{m}\right), y, \ldots, y^{m-1}\right\}$.

Case 2. $c c^{\prime}=0$ : Then since $\eta \nsim *$ we have either (a) $D z=x y, D z^{\prime}=x^{l}$ or (b) $D z=x y, D z^{\prime}=y^{m}$. Therefore, we have (i)'-(iv) ${ }^{\prime}$ as follows.

(i) ${ }^{\prime}$ and (ii)' They are similar as (i) and (ii) but there is no relation $\lambda^{l}=\mu^{m}$.

(iii) $)^{\prime}$ For $(\mathrm{a})$, there is an element $\left[z x^{l-1}+y z^{\prime}\right]$ in $H^{*}(X ; \mathbb{Q})$. For $(\mathrm{b})$, there is an element $\left[z y^{m-1}+x z^{\prime}\right]$ in $H^{*}(X ; \mathbb{Q})$. In both cases, $X$ are not formal by Theorem 4.1 of $[3]$.

$(\text { iv) })^{\prime}$ There are bases in cohomology $\left\{\left[y^{i}\right]\right\}_{i=0}^{\infty}$ for (a) and $\left\{\left[x^{i}\right]\right\}_{i=0}^{\infty}$ for (b).

Example 4.11. Let $X$ be the homotopy fibre of a map $f: Y=K(\mathbb{Q}, 4) \vee K(\mathbb{Q}, 6) \rightarrow$ $K(\mathbb{Q}, 12)$ defined by $f^{*}\left(\iota_{12}\right)=\iota_{4}^{3}+\iota_{6}^{2} \in H^{12}(Y)$, where $\iota_{4}, \iota_{6}, \iota_{12}$ are generators of the rational cohomology groups. Let $X^{\prime}$ be the homotopy fibre of a map $g: Y=$ $K(\mathbb{Q}, 4) \vee K(\mathbb{Q}, 6) \rightarrow K(\mathbb{Q}, 12)$ defined by $g^{*}\left(\iota_{12}\right)=\iota_{6}^{2}$. We see

$$
M(X)=\left(\Lambda\left(x, y, z, z^{\prime}\right), D\right) \text { and } M\left(X^{\prime}\right)=\left(\Lambda\left(x, y, z, z^{\prime}\right), D^{\prime}\right)
$$

with $|x|=4,|y|=6,|z|=9,\left|z^{\prime}\right|=11, D x=D y=0, D z=x y$ and $D z^{\prime}=x^{3}+y^{2}$; $D^{\prime} x=D^{\prime} y=0, D^{\prime} z=x y$ and $D^{\prime} z^{\prime}=y^{2}$. Then $\pi_{*}(\Omega X) \cong \pi_{*}\left(\Omega X^{\prime}\right)$ as rational homotopy Lie algebras. But, we have

$$
N \mathcal{E}(X)=4<N \mathcal{E}\left(X^{\prime}\right)=N \mathcal{E}(Y)=6 .
$$

The reader is referred to Proposition on p. 88 of [17], Proposition 13.16 and Theorem 21.6 of [4] or pp. $152-154$ of [8] for the relation between the rational Whitehead product and the quadratic part of the differential of the Sullivan model.

\section{References}

[1] C. Allday and S. Halperin, Lie group actions on spaces of finite rank, Quart. J. Math. Oxford Ser. (2) 29 (1978), 63-76.

[2] H.W. Choi and K.Y. Lee, Certain numbers on the groups of self-homotopy equivalences, Topology Appl. 181 (2015), 104-111.

[3] P. Deligne, P. Griffiths, J. Morgan and D. Sullivan, Real homotopy theory of Kähler manifolds, Invent. Math. 29 (3) (1975), 245-274.

[4] Y. Félix, S. Halperin and J.-C. Thomas, Rational Homotopy Theory, Grad. Texts in Math. 205, Springer-Verlag, New York, Heidelberg, Berlin, 2001.

[5] Y. Félix, J. Oprea and D. Tanré, Algebraic Models in Geometry, Oxf. Grad. Texts Math. 17, Oxford University Press, Oxford, 2008.

[6] T. Ganea, A generalization of the homology and homotopy suspension, Comment. Math. Helv. 39 (1965), 295-322.

[7] B. Gray, Homotopy Theory. An Introduction to Algebraic Topology, Pure Appl. Math. 64, Academic Press, New York, London, 1975.

[8] P.A. Griffiths and J.W. Morgan, Rational Homotopy Theory and Differential Forms, Progr. Math. 16, Birkhäuser, Boston, 1981.

[9] S. Halperin, Rational fibrations, minimal models, and fibrings of homogeneous spaces, Trans. Amer. Math. Soc. 244 (1978), 199-224. 
[10] S. Halperin, Rational Homotopy and Torus Actions, Aspects of topology, 293306, London Math. Soc. Lecture Note Ser. 93, Cambridge Univ. Press (1985).

[11] S. Halperin and J. Stasheff, Obstructions to homotopy equivalences, Adv. Math. 32 (1979), 233-279.

[12] P. Hilton, G. Mislin and J. Roitberg, Localization of Nilpotent Groups and Spaces, North-Holland Math. Stud. 15, North-Holland Publishing Co., Amsterdam, Oxford, American Elsevier Publishing Co., New York, 1975.

[13] J.R. Munkres, Elements of Algebraic Topology, Addison-Wesley Publishing Company, Menlo Park, CA, 1984.

[14] Y. Nomura, Homotopy equivalences in a principal fibre space, Math. Z. 92 (1966), 380-388.

[15] N. Oda and T. Yamaguchi, Self-homotopy equivalences and cofibrations, Topology Appl. 228 (2017), 341-354.

[16] D. Sullivan, Infinitesimal computations in topology, Publ. Math. Inst. Hautes Études Sci. 47 (1978), 269-331.

[17] D. Tanré, Homotopie rationnelle: modèles de Chen, Quillen, Sullivan, Lecture Notes in Math. 1025, Springer-Verlag, Berlin, Heidelberg, 1983.

[18] G.W. Whitehead, Elements of Homotopy Theory, Grad. Texts in Math. 61, Springer-Verlag, New York, Heidelberg, Berlin, 1978.

Nobuyuki Oda odanobu@cis.fukuoka-u.ac.jp

Department of Applied Mathematics, Faculty of Science, Fukuoka University, Fukuoka 814-0180, Japan

Toshihiro Yamaguchi tyamag@kochi-u.ac.jp

Faculty of Education, Kochi University, 2-5-1 Akebono-cho, Kochi 780-8520, Japan 